OPEN ACCESS

Edited by:

Takaki Maekawa

Max Planck Institute for Plant

Breeding Research, Germany

Reviewed by:

Brian Staskawicz,

University of California, Berkeley,

United States

Xin Li,

The University of British Columbia,

Canada

${ }^{*}$ Correspondence:

Marc T. Nishimura

marcusn@colostate.edu

Specialty section:

This article was submitted to Evolutionary and Population Genetics,

a section of the journal

Frontiers in Genetics

Received: 16 February 2020

Accepted: 04 May 2020

Published: 02 June 2020

Citation:

Bayless AM and Nishimura MT (2020) Enzymatic Functions for Toll/Interleukin-1 Receptor Domain Proteins in the Plant Immune System.

Front. Genet. 11:539.

doi: $10.3389 /$ fgene.2020.00539

\section{Enzymatic Functions for Toll/Interleukin-1 Receptor Domain Proteins in the Plant Immune System}

\author{
Adam M. Bayless and Marc T. Nishimura* \\ Department of Biology, Colorado State University, Fort Collins, CO, United States
}

Rationally engineered improvements to crop plants will be needed to keep pace with increasing demands placed on agricultural systems by population growth and climate change. Engineering of plant immune systems provides an opportunity to increase yields by limiting losses to pathogens. Intracellular immune receptors are commonly used as agricultural disease resistance traits. Despite their importance, how intracellular immune receptors confer disease resistance is still unknown. One major class of immune receptors in dicots contains a Toll/Interleukin-1 Receptor (TIR) domain. The mechanisms of TIR-containing proteins during plant immunity have remained elusive. The TIR domain is an ancient module found in archaeal, bacterial and eukaryotic proteins. In animals, TIR domains serve a structural role by generating innate immune signaling complexes. The unusual animal TIR-protein, SARM1, was recently discovered to function instead as an enzyme that depletes cellular $\mathrm{NAD}^{+}$(nicotinamide adenine dinucleotide) to trigger axonal cell death. Two recent reports have found that plant TIR proteins also have the ability to cleave $\mathrm{NAD}^{+}$. This presents a new paradigm from which to consider how plant TIR immune receptors function. Here, we will review recent reports of the structure and function of TIR-domain containing proteins. Intriguingly, it appears that TIR proteins in all kingdoms may use similar enzymatic mechanisms in a variety of cell death and immune pathways. We will also discuss TIR structure-function hypotheses in light of the recent publication of the ZAR1 resistosome structure. Finally, we will explore the evolutionary context of plant TIR-containing proteins and their downstream signaling components across phylogenies and the functional implications of these findings.

Keywords: Toll/interleukin-1 receptor, TIR, NLR, NADase, innate immunity

\section{THE PLANT IMMUNE SYSTEM}

Single and multicellular organisms have evolved numerous defenses to ward off biotic challenges. The plant innate immune system consists of receptor proteins that monitor both extracellular and intracellular pathogen-related signals to activate defenses (Figure 1). Typically, extracellular signals are transduced across the plasma membrane by an extensive array of receptor-like kinase (RLK) and receptor-like proteins (RLPs) (Boutrot and Zipfel, 2017; Tang et al., 2017). Disease resistance conferred by the RLK/RLP pattern recognition receptor (PRR) system is triggered by a wide array of apoplastic molecules from microbes, pathogens and host damage signals. Accordingly, pathogens have evolved to extensively target PRR pathways to promote host susceptibility. A common strategy of plant pathogens is to deliver intracellular 


\section{Plant Cell}

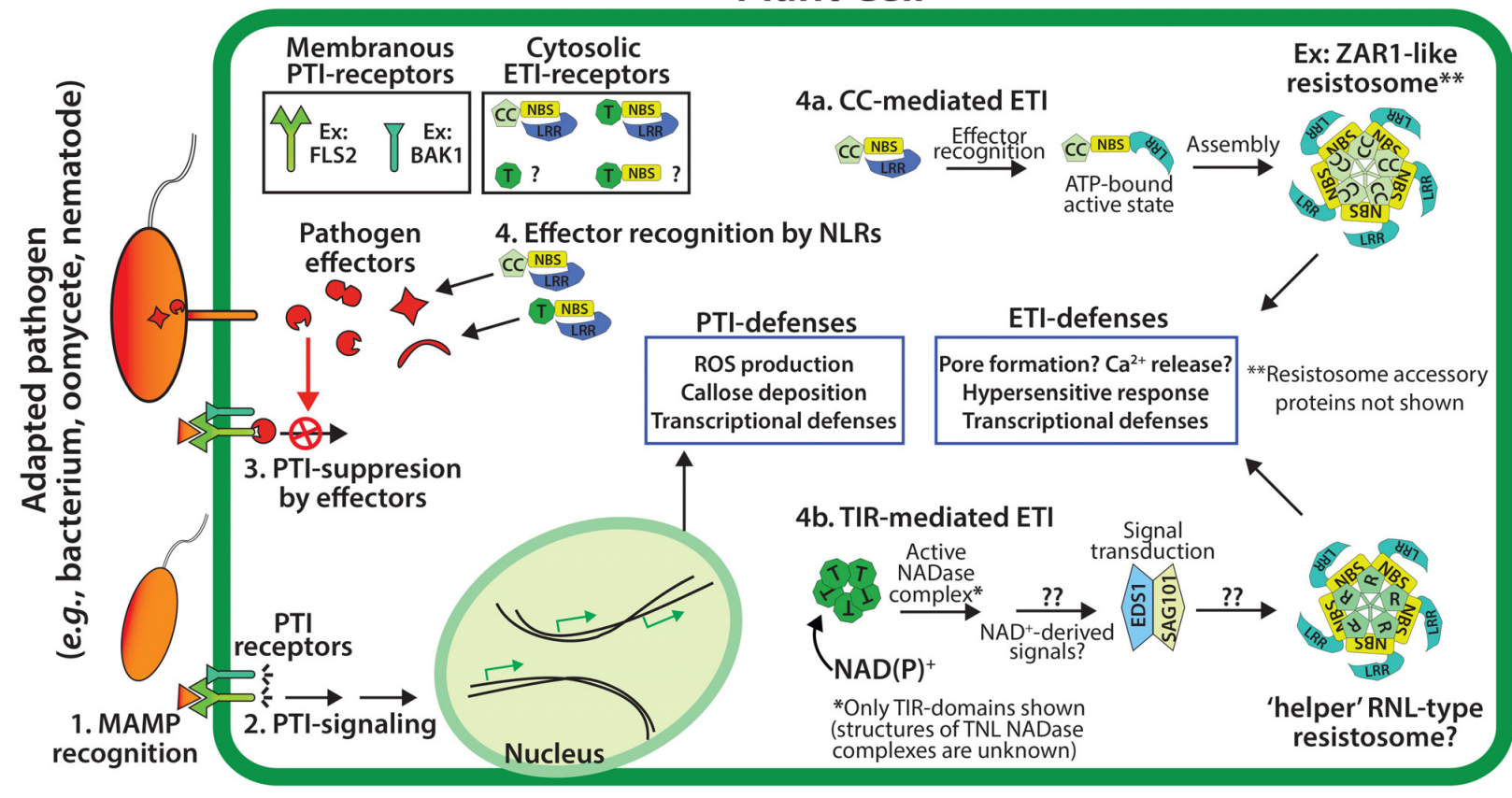

FIGURE 1 | Overview of the two tiers of the plant immune system. PTI (pattern-triggered immunity) utilizes membrane associated PTI receptors to detect conserved microbial associated molecular patterns (MAMPs, e.g., chitin or flagellin), and signal downstream PTI-immunity (steps $\mathbf{1}$ and 2). The effectors of adapted pathogens can disarm PTI-immunity (step 3), while genetically resistant plants utilize NLR-type (NBS-LRR) resistance proteins to detect effector activities and trigger ETI (effector-triggered immunity, step 4), which often includes localized cell death - the hypersensitive response (HR). Plant NLR resistance proteins generally possess $\mathrm{N}$-terminal CC (coiled-coil) or TIR (Toll/interleukin-1 Receptor) domains.

virulence proteins (such as type III effector proteins) in order to disrupt PRR-based defense (Jones and Dangl, 2006; Dangl et al., 2013). These virulence proteins are necessary for pathogenicity, and thus serve as reliable indicators of pathogen presence. In response to pathogen immunosuppression, plants have evolved a second layer of innate immune receptors that directly or indirectly recognize the presence of pathogen virulence proteins (Jones and Dangl, 2006; Qi and Innes, 2013). As such, virulence proteins are the tools that pathogens use to suppress the host immune system, but also the signals that plants of the correct genotype (i.e., resistant plants) can recognize to reinitiate a defense response. These intracellular receptors are characterized by nucleotide-binding site (NBS) domains and a C-terminal Leucine-rich repeat (LRR). This combination of domains is present in both plant and animal NLR proteins (confusingly referring to both "NBS-LRR" and "Nod-like receptors (Nod: $\mathrm{N}$-terminal oligomerization domain)." While plant and animal NLR proteins are functionally conserved in many ways, it appears that they are the product of convergent evolution (Urbach and Ausubel, 2017).

The recognition of intracellular pathogen virulence molecules promotes conformational changes in NLR proteins (Takken and Goverse, 2012). The N-terminal domain of NLR proteins has signaling activities, while the C-terminal NBS-LRR domains negatively regulate signaling in the resting state. The NBS domain functions as a molecular switch depending on the bound nucleotide: ADP-bound in the resting state and ATP-bound in the active state (Takken and Goverse, 2012). Both plant and animal NLRs are auto-regulated and self-associate during signal transduction, however, the N-terminal signaling domains of plant and animal NLRs are distinct (Qi and Innes, 2013; Hu et al., 2015; Nanson et al., 2019). Generally, plant NLRs contain N-terminal TIR (Toll/Interleukin Receptor-1) or CC (coiled coil) domains, and are therefore known as TNLs or CNLs (Qi and Innes, 2013). Monocot genomes appear to lack TNL loci, however, both monocots and dicots can encode TIRonly and TIR-NBS proteins (Meyers et al., 2002; Collier et al., 2011; Nandety et al., 2013; Nishimura et al., 2017; Gao et al., 2018). TIR and CC-domains from plant NLRs are sufficient to activate immune outputs, including a localized cell death termed the hypersensitive response (HR), and transcriptional defense programs (Swiderski et al., 2009; Collier et al., 2011). The selfassociation and oligomerization of either TIR or CC-domains is required for plant immune signaling, however, the downstream events which follow the activation of TIR or CC resistance proteins has remained unclear (Casey et al., 2016; Wan et al., 2019; Wang et al., 2019a).

\section{DOWNSTREAM COMPONENTS OF TIR-SIGNALING PATHWAYS IN PLANTS}

Genetic screens have identified two families of proteins that appear universally required for plant TIR phenotypes 
A

Downstream mediators of plant TIR-signaling

EDS1-family

$\underline{\text { (lipase-like proteins) }}$

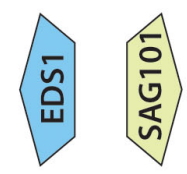

RPW8-type

'helper' NLRs (CNLs)

ADR1: $R \underset{\text { NRS }}{\mathrm{NBS}}$

NRG 1: $R \underset{\text { LRR }}{\text { NBS }}$

B

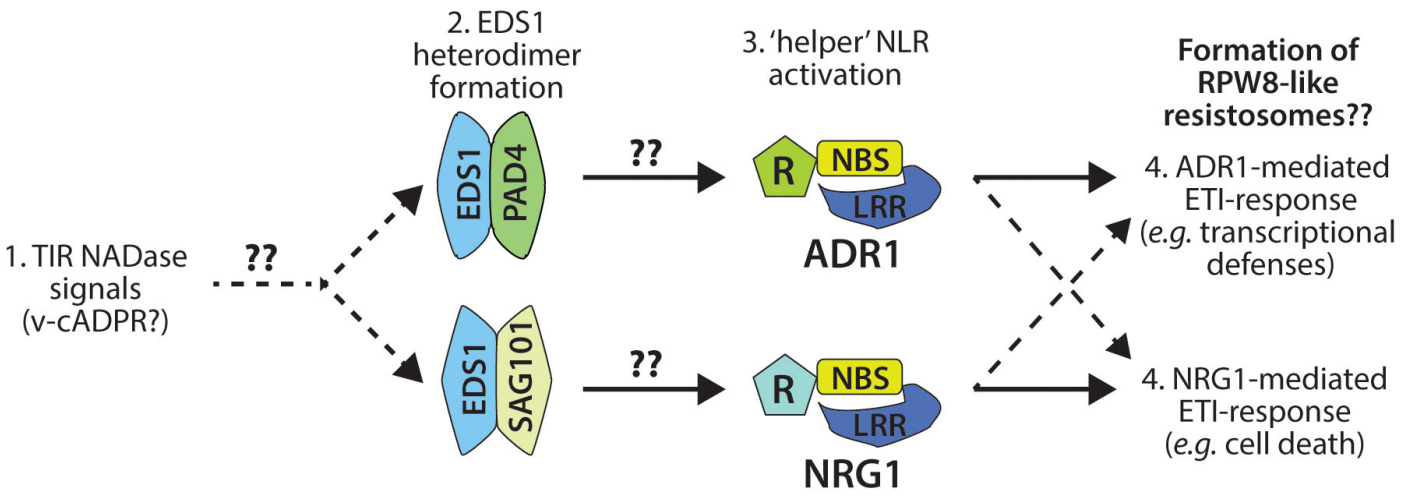

c

TIR-domains in plants and plant-relatives

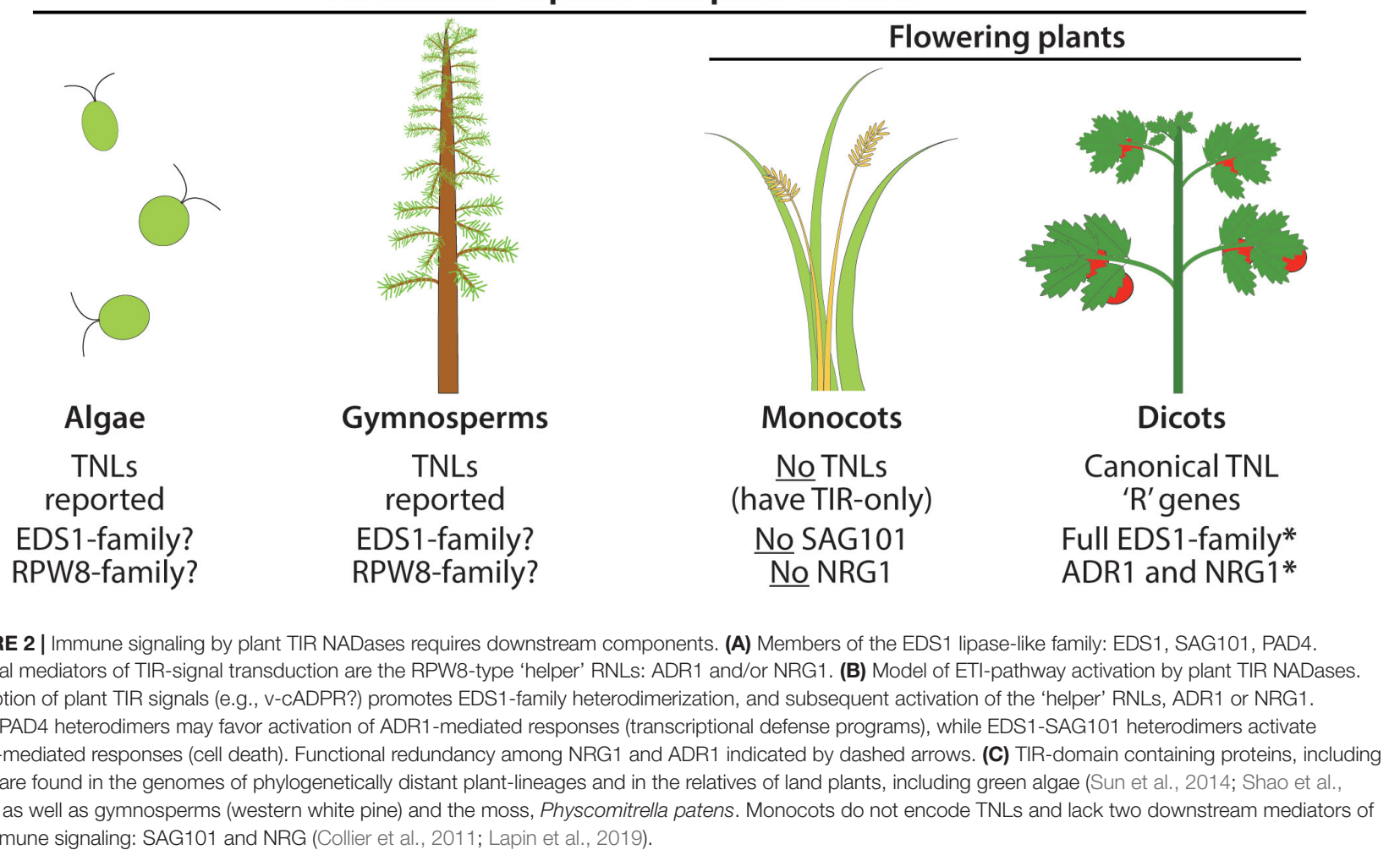

(Figure 2). The first component is the EDS1 (Enhanced Disease Susceptibility 1) family of lipase-like proteins [EDS1, SAG101 (Senescence-Associated Gene 101), and PAD4 (Phytoalexin
Deficient 4)] (Feys et al., 2005; Lapin et al., 2019). The second component, the RPW8 class of 'helper' CNLs, often referred to as 'RNLs', functions downstream of the EDS1 family (Peart et al., 
2005; Qi et al., 2018; Jubic et al., 2019; Wu et al., 2019). Helper NLRs such as the NRG1 (N-requirement Gene 1) and the ADR1 family (Activated Disease Resistance 1) are candidates for being the ultimate output of TIR pathways (Collier et al., 2011; Qi et al., 2018; Jubic et al., 2019). How these downstream components are activated by TIR oligomerization, and the organization of the overall pathway, remains a major unanswered question (Jubic et al., 2019; Lapin et al., 2019; Wan et al., 2019).

EDS1 forms exclusive heterodimers with either PAD4 or SAG101 to relay TIR-immune signals (Feys et al., 2005; Wagner et al., 2013). EDS1 and PAD4 are also reported to function in plant basal defenses and salicylic acid signaling (Cui et al., 2017). The crystal structure of the EDS1-SAG101 heterodimer suggests that binding of the $\mathrm{N}$-terminal lipase-like domains establishes unique interaction interfaces at the C-terminal EP domain (Wagner et al., 2013). The C-terminal EP-domain of EDS1-members contains positively charged residues and is essential for transduction of TIR-signals (Bhandari et al., 2019; Lapin et al., 2019). The TNL RPS4 (Resistance to Pseudomonas syringae 4), as well as particular TIR-NBS proteins, have been reported to associate with EDS1, as has the 'helper' NLR, NRG1 (Heidrich et al., 2011; Nandety et al., 2013; Huh et al., 2017; Qi et al., 2018). The functional consequences of these physical interactions are unknown. Lapin et al. (2019) determined that the EDS1-members of Solanaceous species could complement a $N$. benthamiana mutant which lacks all EDS1family members. However, the orthologous EDS1-members of Arabidopsis did not complement, suggesting that within species, EDS1-members may have co-evolved a high degree of specificity in the relay of TIR-signals (Lapin et al., 2019). Curiously, in the absence of downstream 'helper' NLRs, EDS1-members can still mediate limited transcriptional defense programs from an auto-active version of the TNL, Roq1 (Recognition of XopQ1) (Qi et al., 2018).

The expression of the RPW8-domains of ADR1 or NRG1 is sufficient to trigger $\mathrm{HR}$, even in eds1 null backgrounds, placing 'helper' RNLs as downstream mediators of TIR-signaling (Collier et al., 2011; Qi et al., 2018). Typically, plant genomes carry relatively few loci encoding helper RNLs, consistent with a conserved RNL function that integrates inputs channeled from upstream TNL receptors via EDS1-complexes. Additionally, functional redundancy between ADR1 and NRG1 has been reported (Castel et al., 2019; Jubic et al., 2019; Lapin et al., 2019; Wu et al., 2019). Some CNLs are also reported to signal through ADR1, suggesting that cross-talk might occur at the endpoints of certain CNL and TNL-signal pathways (Castel et al., 2019; Wu et al., 2019). The RPW8-domain of helper RNLs does share similarities with the CC-domain of CNLs; thus, the recent structure of the ZAR1 (HOPZ-ACTIVATED RESISTANCE 1, a $\mathrm{CNL}$ ) resistosome may provide insights into the functions of the ADR1 and NRG1 helper NLRs (Wang et al., 2019a,b). The active ZAR1 complex assembles into a ring-shaped pentamer, the "resistosome", and hypothetically disrupts cell membrane integrity with a pore-forming channel (Wang et al., 2019a,b).

The mechanisms of how plant NLRs activate downstream immunity is an active area of research. While TIR-TIR interactions are well known to promote animal immune signaling via scaffold function, a new paradigm of plant TIR function has recently emerged: signal competent plant TIR-domains are $\mathrm{NAD}^{+}$-(nicotinamide adenine dinucleotide)-hydrolyzing enzymes (Figures 3A-D) (Horsefield et al., 2019; Wan et al., 2019). Below, we review recent advances in the understanding of plant TIR-domain structure, evolution, and enzymatic (NADase) function. We also draw insights from the TIR-NADases encoded by animals and prokaryotes, and explore how the newly reported structure of the ZAR1 CNL 'resistosome' complex might inform the high order complexes of plant TIR-NADases.

\section{TIR-DOMAINS: A CELLULAR DEFENSE MODULE FOUND IN ALL DOMAINS OF LIFE}

Toll/Interleukin Receptor-1 (TIR)-domain containing proteins are found in all domains of life - Eukarya, Bacteria, and Archaea (Figures 4A,B) (Essuman et al., 2018). Frequently, TIR-domain containing proteins function in immunity or cell death decisions in bacteria, plants and animals, suggesting an ancient role in cellular defenses (Figures 3A,B, 4A,B). The core TIR-domain is typically $\sim 120-200$ residues, and is found in multi-domain and single domain proteins (Nimma et al., 2017). TIR-domains generally require TIR-TIR self-associations for function, and TIRdomains can also participate in heterotypic protein interactions. The sequence identity of TIR-domains among different species may be as low as 20-30\%, however, TIR-domains share a flavodoxin-like fold, consisting of parallel beta-sheets and alphahelices with interconnecting loops (Ve et al., 2015).

\section{INSIGHTS TO PLANT TIR FUNCTION FROM ANIMAL SYSTEMS: SARM1 (STERILE ALPHA AND TIR MOTIF-CONTAINING 1) IS AN NADase}

Typically, animal TIRs (e.g., Toll-like receptors, MyD88) couple pathogen detection to defense gene activation by nucleating the formation of large multimeric signaling complexes (Figure 3A) (Xu et al., 2000; O’Neill and Bowie, 2007; Kenny and O'Neill, 2008; Nimma et al., 2017). Crystal structures for numerous animal TIR-domains have acted as guides for a biochemical dissection of TIR-domain function (Xu et al., 2000; Valkov et al., 2011; Bovijn et al., 2012). The crystal structure of the TIRdomain from Toll Like Receptor 2 (TLR2) revealed residues required for TIR-TIR interactions, and the core TIR-domain structure of parallel beta-sheets and alpha-helices (Xu et al., 2000). Additional structural studies of TIR-adaptor proteins further defined TIR interfaces required for multimerization and signal complex formation (Nyman et al., 2008; Valkov et al., 2011; Bovijn et al., 2012). Animal TIR scaffolding can signal various defensive outputs, such as inflammatory responses and cytokine production (Figure 2A) (O'Neill and Bowie, 2007). In contrast, the unusual animal TIR protein SARM1 (sterile alpha and TIR motif-containing 1) was recently found to have a surprising enzymatic function (Essuman et al., 2017). 
A TIR-scaffolds (animals)

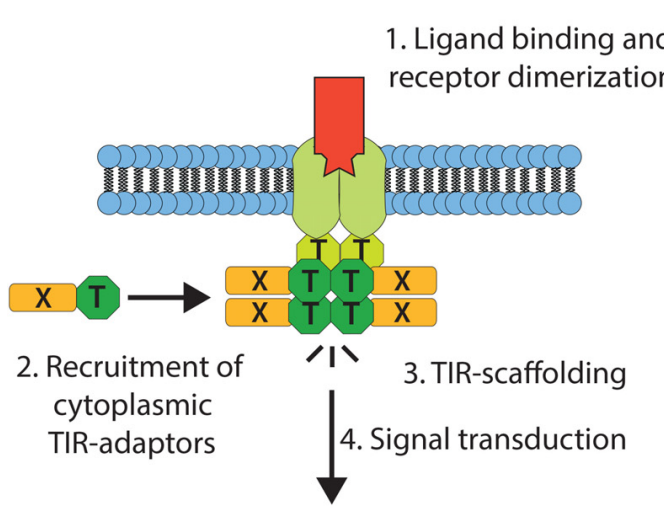

5. Innate immune response (e.g., inflammatory response)

\section{B TIR-NADases (animals/plants)}

\author{
2. Activated \\ SARM1-NADase \\ Complex
}

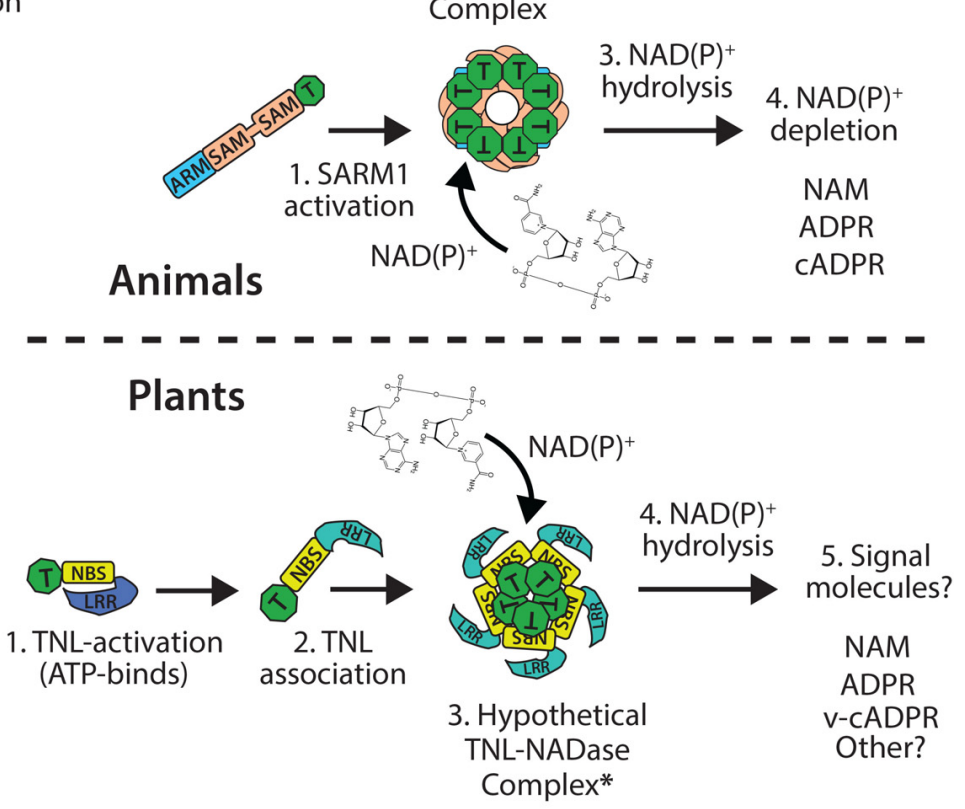

c

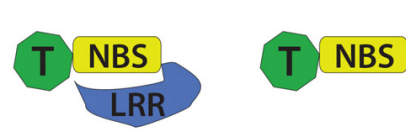

Examples of TIR-domain containing proteins
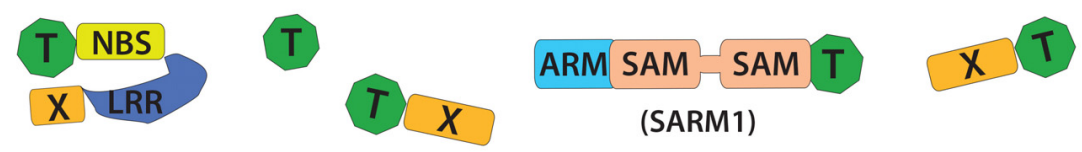

(SARM1)

D

Known enzymatic products of TIR-domain NADases

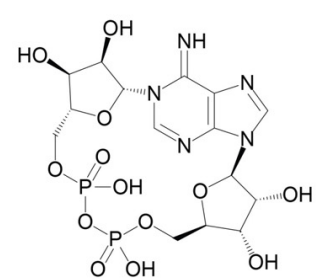

cyclic-ADPR (cADPR)

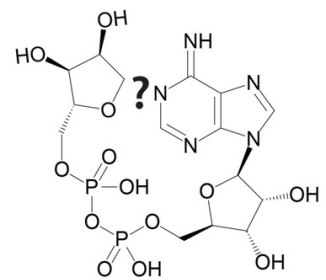

variant

cyclic-ADPR

( $v$-CADPR)<smiles>NC(=O)c1cccnc1</smiles>

Nicotinamide (NAM)

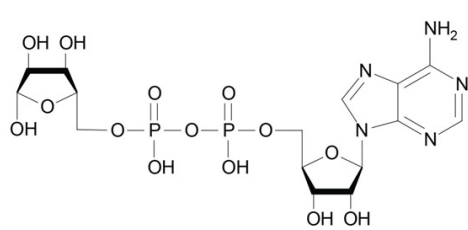

ADP-Ribose (ADPR)

FIGURE 3 | Model of TIR-domain scaffolding (animals) and TIR-NADase activity (plants and animals). (A) Canonical TIR-scaffold function in animals: TIR-TIR interactions promote signal complex formation and innate immune signal transduction. (B) Top: animal TIR NADases (e.g., SARM1) assemble into high order complexes, and hydrolyze $\mathrm{NAD}(\mathrm{P})^{+}$substrate and alter $\mathrm{NAD}(\mathrm{P})^{+}$pools. Bottom: assembly of plant TIR-domains into hypothetical NADase complex (resistosome-like?) and generation of immunomodulatory signals. (C) Numerous TIR-domain configurations are present in animal, plant, and bacterial proteins. Plant TIR-domains are often found in modular NBS-LRRs, TIR-NBS, TIR-X or TIR-only proteins. -X corresponds to atypical or undefined domains. The animal SARM1 TIR is located at the C-terminus; the SARM1 SAM-domains promote oligomerization. (D) Known products of TIR NADases; plant TIRs produce variant cyclic-ADPR (v-cADPR), whose structure is currently unknown.

The animal TIR protein SARM1 functions in axon degeneration, an active process of programmed cell death in response to injury (classically known as "Wallerian degeneration") (Gerdts et al., 2015). $\mathrm{NAD}^{+}$-depletion had been associated with axon degeneration, but the SARM1regulated NADase had remained elusive. The critical observation that the TIR domain of SARM1 is structurally similar to bacterial nucleotide-processing enzymes led to the 


\section{A TIRs in animals}

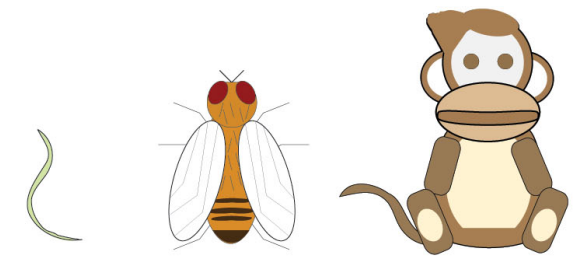

1. TIR scaffolds (non-enzymatic) -innate immune complexes

\section{TIR NADases (i.e., SARM1) \\ -axon degeneration \\ -innate immunity? \\ $-\mathrm{Ca}^{2+}$ signaling?}

\section{TIRs in prokaryotes}

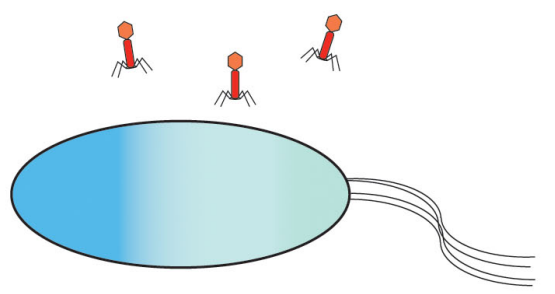

1. Anti-phage immunity

-Theoris and cBASS variants

\section{Virulence factors \\ -disrupt host physiology}

FIGURE 4 | TIR NADases were recently reported in animals and prokaryotes. (A) Diverse eukaryotic organisms, including invertebrates (e.g., C. elegans, D. melanogaster) and vertebrates, utilize TIR-domain containing proteins in cellular innate immunity. Non-enzymatic TIR-domain containing proteins in animals promote signal complex formation via TIR - TIR interactions. The SARM1 NADase TIR from animals functions in axon degeneration, and is reported to function in immunity in C. elegans (Shivers et al., 2009). (B) Numerous bacteria and archaeal species encode TIR-NADases. Prokaryotic TIR-domain containing proteins are reported to function in anti-phage immunity (Thoeris system and variants of cBASS) (Doron et al., 2018; Cohen et al., 2019). TIR-domains from pathogenic bacteria are reported to function in virulence (Alaidarous et al., 2014).

recognition that the SARM1 TIR has an intrinsic enzymatic activity: $\mathrm{NAD}^{+}$-hydrolase function (Figure 3B) (Gerdts et al., 2015; Essuman et al., 2017). Axon degeneration requires SARM1 TIR domain NADase activities (Essuman et al., 2017). The unusual enzymatic activity of SARM1 TIR relative to other animal TIR domains is perhaps reflected in an unusual evolutionary history, as the SARM1 TIR appears to have been horizontally transferred into animals (Zhang et al., 2011). TIRs that function in canonical TLR pathways (TLR4 and MyD88) do not have NADase activity, although the family has not been exhaustively tested (Essuman et al., 2017).

Like NLRs, SARM1 is a multidomain TIR protein that is autoinhibited. SARM1 has two tandem sterile alpha (SAM) domains, which enable oligomerization, and an N-terminal Armadillo domain, which is required for auto-inhibition (Figures 3B,C) (Essuman et al., 2018). SARM1 TIR NADase function is dependent upon oligomerization and TIR-TIR associations. The mechanism of activation during axon degeneration is unclear, but NADase activity of SARM1 can be enhanced by phosphorylation or treatment with a cell-permeant mimetic of nicotinamide mononucleotide, an $\mathrm{NAD}^{+}$precursor (Murata et al., 2018; Zhao et al., 2019).

$\mathrm{NAD}^{+}$-hydrolysis by SARM1 generates ADPR (ADP-ribose), cyclic ADPR (c-ADPR) and NAM (nicotinamide) (Essuman et al., 2017) (see Figure 3D). The products of SARM1mediated $\mathrm{NAD}^{+}$-hydrolysis (cADPR, ADPR) are known $\mathrm{Ca}^{2+}$ mobilization agents and may thus effect cellular $\mathrm{Ca}^{2+}$ signaling (Lee, 2012; Guse, 2015; Lee and Zhao, 2019; Zhao et al., 2019). SARM1 readily hydrolyzes $\mathrm{NADP}^{+}$as well as NADanalogs with substitutions to the adenine ring, such as amino group additions (Essuman et al., 2017). However, FAD (flavin adenine dinucleotide) and NADH or NAD-analogs lacking the amino group of the nicotinamide ring could not be hydrolyzed (Essuman et al., 2017, 2018). Depending on local cellular pH, SARM1 is also reported to generate NAAD (nicotinic acid adenine dinucleotide) (Zhao et al., 2019).

A recent crystal structure of the SARM1 TIR reveals conservation with both plant and prokaryotic TIR-domains (Horsefield et al., 2019). The active site of the SARM TIRdomain includes a conserved glutamic acid (E642) which is required for $\mathrm{NAD}^{+}$-hydrolysis (Figure 5A). Recent crystal and cryo-EM structures of SARM1 complexes, and of the tandem SAM-domains, indicate that the active SARM1 NADase complex forms a ring-shaped octamer (Horsefield et al., 2019; Sporny et al., 2019) (Figure 5B). The crystal structure of the SARM1 TIR active site revealed close proximity of ribose with the putative catalytic glutamate (E642) and may suggest potential substrateactive site interactions (Figure 5C) (Horsefield et al., 2019). The exact catalytic mechanism of SARM1 is unknown, but appears distinct from $\mathrm{CD} 38$, which also produces cADPR from $\mathrm{NAD}^{+}$ (Loring et al., 2020).

Strikingly, SARM1 triggers cell death when transiently expressed in the leaves of the plant, Nicotiana benthamiana (Horsefield et al., 2019; Wan et al., 2019). Like axon degeneration, plant cell death triggered by SARM1 requires NADase function, however, SARM1-mediated cell death occurs independently of the known plant TIR-signaling components EDS1 and NRG1 (Horsefield et al., 2019; Wan et al., 2019). Notably, supplementation of exogenous $\mathrm{NAD}^{+}$reduces axon degeneration mediated by SARM1 (Gerdts et al., 2015). As such, SARM1 depletion of cellular $\mathrm{NAD}(\mathrm{P})^{+}$is likely to underlie both animal 
A

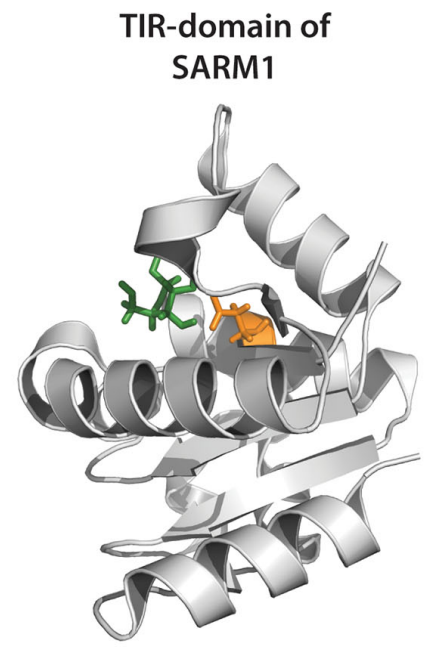

PDB ID: $600 Q$
B

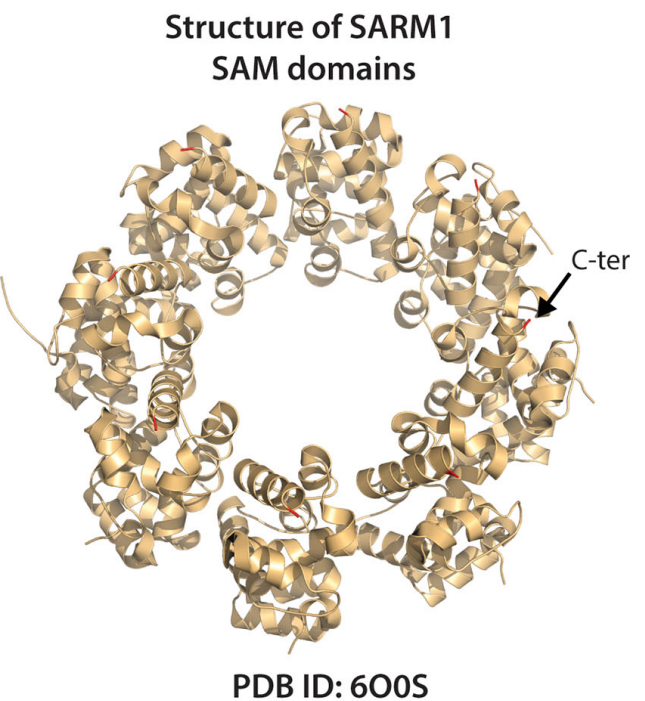

SARM1-TIR

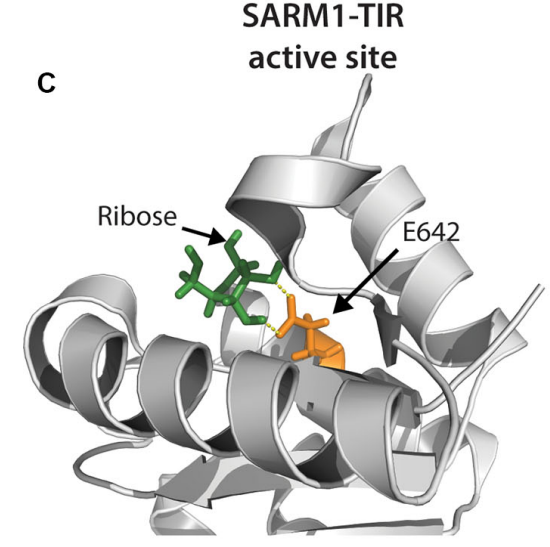

PDB ID: $600 Q$

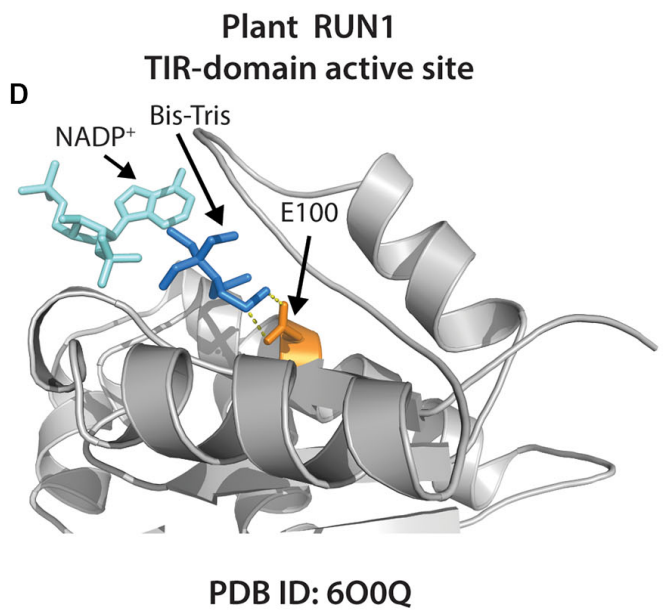

FIGURE 5 | Structures of individual animal and plant TIR-domain NADases, and the higher order SARM1 SAM octamer. (A) Crystal structure of the SARM1-TIR domain (PDB ID: 6O0Q) with ribose (shown green) positioned near putative catalytic glutamate residue (E642), colored orange. (B) Crystal structure of tandem SAM domains of the animal TIR-NADase, SARM1 (PDB ID: 6O0S). The SARM1 SAM domains adopt a closed octameric ring conformation. C-terminal end of SAM tipped with red (arrow shown for one unit). (C) Close-up view of SARM1 TIR active site, as in (A). Arrows indicate ribose and putative catalytic E642 (ribose $\sim 2.6 \AA$ from E642). (D) Close-up of active site of the TIR-domain from plant TNL, RUN1 (PDB ID: 6O0W). A bis-Tris molecule (dark blue) positioned near putative catalytic glutamate (E100, orange) precludes access of $\mathrm{NADP}^{+}$-substrate (aqua); bis-Tris $\sim 3 \AA$ from $\mathrm{E} 100$.

axon degeneration and plant cell death resulting from its ectopic expression. However, some cell lines are reported to tolerate low levels of SARM1 expression (Lee and Zhao, 2019; Zhao et al., 2019). Whether low level SARM1 activity in particular cellular contexts might generate signaling molecules vs. deplete cellular $\mathrm{NAD}^{+}$stores, is not yet clear.

\section{TIR NADases IN PROKARYOTES: PHAGE IMMUNE SYSTEMS AND VIRULENCE FACTORS}

Numerous bacterial and archaeal species encode TIR-domain containing proteins, primarily of unknown function (Spear et al.,
2009; Doron et al., 2018; Essuman et al., 2018). However, some prokaryotic TIRs are reported to function in anti-phage immunity, while other TIRs may act as virulence factors which manipulate host responses (Figure 4B) (Alaidarous et al., 2014; Doron et al., 2018; Coronas-Serna et al., 2019). TIRdomains encoded by Brucella and Paracoccus are reported to mimic animal TIR-adaptors and disrupt TLR immune signaling, potentially via physical interactions with animal TIR domains (Chan et al., 2009; Alaidarous et al., 2014; Snyder et al., 2014). However, many apparently non-pathogenic bacteria encode TIR-proteins, suggesting that some TIR-domains could possess functions outside of virulence or immunity (Spear et al., 2009). $\mathrm{NAD}^{+}$-hydrolase activities have recently been shown for several bacterial and archaeal TIRs, and 
thus, it has been suggested that ancestrally, the TIR-domain belongs to a large family of nucleotide hydrolase enzymes (Essuman et al., 2018).

Like the SARM1 TIR NADase, all examined prokaryotic TIRs also require the putative catalytic glutamate for NADase function (Essuman et al., 2018). Prokaryotic TIRs are likely to also require TIR-TIR self-associations, as local crowding (via TIR protein laden beads) enhanced NADase function (Essuman et al., 2018). Prokaryotic TIR-domains show variation in terms of $\mathrm{NAD}^{+}$-hydrolysis kinetics, as well as in the type and ratio of products produced from $\mathrm{NAD}^{+}$-hydrolysis (Essuman et al., 2017). For example, the TirS TIR domain from Staphylococcus aureus generated ADPR and CADPR, while the TcpO TIR domain from the archaea Methanobrevibacter olleyae produced a novel product initially termed metabolite $\mathrm{X}$, which is likely a variant of cyclic ADPR ( $\mathrm{v}$-cADPR), whose structure remains unresolved (Essuman et al., 2017; Wan et al., 2019).

Recent studies from the Sorek lab may provide a glimpse into the origins of TIR-mediated immunity (Figure 4B) (Doron et al., 2018; Cohen et al., 2019). A survey of tens of thousands of prokaryotic genomes, coupled with functional screening, unveiled multiple new classes of antiphage defense systems. Among these, an anti-phage system termed Thoeris, was found in $\sim 2,000$ bacterial and archaeal genomes (Doron et al., 2018). The Thoeris defense operon encodes an $\mathrm{NAD}^{+}$binding protein (ThsA) and a TIR-domain protein (ThsB). Both ThsA and B are required for anti-phage immunity. Amino acid alignment of the ThsB TIR-domain with the SARM1-TIR indicated conservation of the catalytic glutamate (Doron et al., 2018). We used Phyre2 to model the B. amyloliquefaciens encoded ThsB ( $B a \mathrm{ThsB}$ ), and retrieved a top-match (60\% identity, $100 \%$ confidence) to the crystal structure (PDB ID: $3 \mathrm{HYN}$ ) of a putative signal transduction factor from Agathobacter rectalis (Figures 6A,B). A comparison of the SARM1 TIR and plant RPS4 TIR structures with the BaThsB TIR-domain model indicates positional conservation of the putative catalytic glutamate (Figure 6A). The putative catalytic glutamate (E99) of ThsB was required for phage protection, suggesting that TIR domains may have an ancient enzymatic-based immune function (Doron et al., 2018). It will be interesting to assess if Thoeris functions via $\mathrm{NAD}^{+}$depletion, akin to SARM1, or could generate $\mathrm{NAD}^{+}$-derived immunomodulatory signals.

The Sorek group further reported that some prokaryote genomes harbor an ortholog of the cGAS-STING defense system found in animals (Cohen et al., 2019). Upon detecting invading DNA, cGAS (cyclic GMP-AMP synthase) generates cyclic GMP-AMP (cGAMP) via oligonucleotide cyclase activity. The cGAMP signal then promotes host cell demise through activating a phospholipase which disrupts membrane integrity (Cohen et al., 2019). This prokaryotic system was dubbed CBASS for cyclic oligonucleotide-based anti-phage signaling system. Notably, variants of CBASS-mediated immunity can encode TIR-domains (Cohen et al., 2019). Whether the TIR-domains of particular CBASS variants require NADase function is uncertain. Nonetheless, it is becoming clear that TIR-mediated immunity to phages is common in both bacteria and archaea. CBASS and Thoeris appear to trigger host cell death prior to the completion of viral replication, thereby restricting phage release into the bacterial population. Elucidating the molecular mechanisms of these prokaryotic TIR-based systems may provide insights into the evolution and function of both immunity and cell death in plants and animals.

\section{TIR NADase ACTIVITY IN PLANTS}

Similar to animal SARM1, plant TIRs were recently demonstrated to be $\mathrm{NAD}^{+}$hydrolases, and this NADase activity is required to relay immune signals (Horsefield et al., 2019; Wan et al., 2019). Sequence analysis of the TIR-domain encoding genes from Arabidopsis, as well as $\sim 8,000$ TIR sequences found from 108 available plant genomes, indicates high conservation ( $\sim 90 \%$ ) of the putative catalytic glutamate required for NADase activity (Wan et al., 2019). The minority of TIR-domains that lack this conserved glutamate appear to be from 'sensortype' TNLs which function via a signal-competent, genomically paired TNL. These sensor-type TNLs lack the ability to trigger cell death or immunity without their partner TNL (Wan et al., 2019).

Like SARM1 of animals, the NADase activity of plant TIRs was required for TIR-domain function; i.e., to relay immune signals (Horsefield et al., 2019; Wan et al., 2019). In vitro NADase cleavage activity was demonstrated by TIR-domains from full length TNLs, as well as TIR-only proteins from dicot plants (Horsefield et al., 2019; Wan et al., 2019). Similar to SARM1 TIR and prokaryotic TIRs, plant TIR-domains could utilize $\mathrm{NAD}^{+}$and $\mathrm{NADP}^{+}$as a substrate, but not the structurally related $\mathrm{NAD}^{+}$precursor NAAD (nicotinic acid adenine dinucleotide) (Essuman et al., 2018; Horsefield et al., 2019; Wan et al., 2019). Intriguingly, a TIR-only protein from the monocot, Brachypodium distachyon (BdTIR), also displayed $\mathrm{NAD}^{+}$-hydrolysis, in addition to triggering an EDS1/NRG1dependent HR, suggesting that TIR-immune signaling may be conserved among dicot and monocot plants (Wan et al., 2019). The products generated by plant TIR NADase reactions include NAM, ADPR, and v-cADPR. Unlike the SARM1 TIR, production of cyclic-ADPR by plant TIRs was not detected. V-cADPR has a near identical HPLC retention time and molecular mass to the product of an archaeal TIR, TcpO (Essuman et al., 2018; Wan et al., 2019).

A crystal structure of the plant TIR-domain, RUN1, with bound $\mathrm{NADP}^{+}$substrate was determined by Horsefield et al. (2019) (Figure 5D). The putative catalytic glutamate of RUN1 was associated with a molecule of bis-Tris, while $\mathrm{NADP}^{+}$was bound near the periphery of the proposed active site (Figure 5D). Accordingly, bis-Tris addition to RUN1 NADase assays inhibited activity, suggesting that bis-Tris association with active site residues may preclude $\mathrm{NADP}^{+}$access and subsequent hydrolysis (Horsefield et al., 2019). How the $\mathrm{NAD}(\mathrm{P})^{+}$substrate interacts with and positions in the active site of plant TIRs during catalysis remains to be determined. 


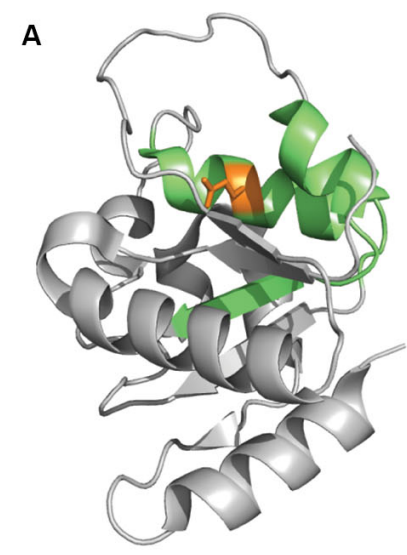

SARM1-TIR (PDB ID: 600Q)

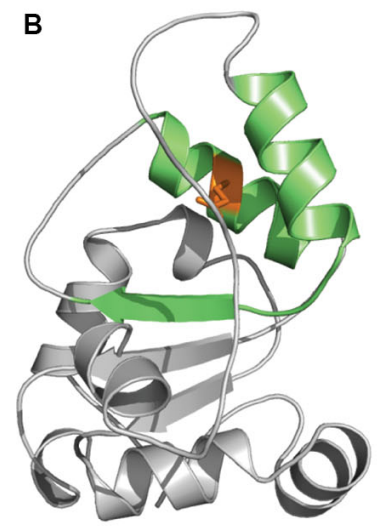

BaThsB-TIR (Modeled to Agathobacter-TIR)
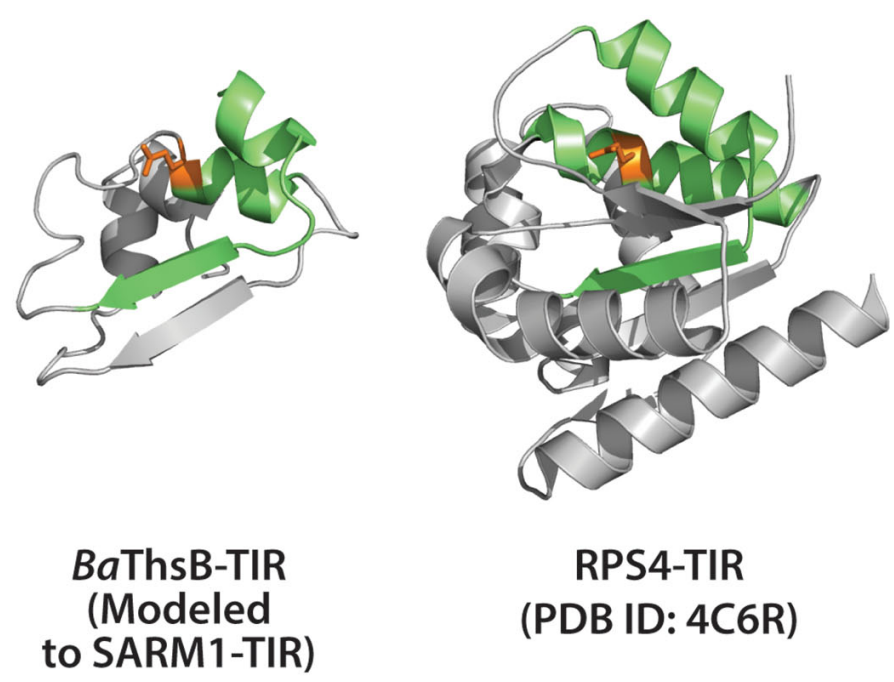
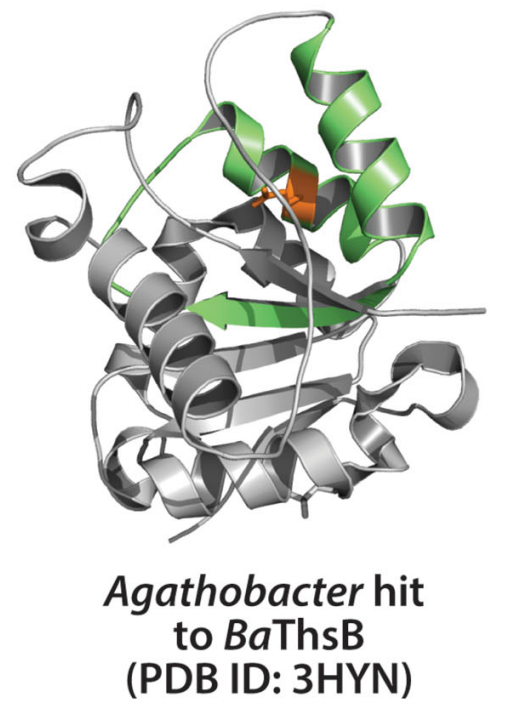

FIGURE 6 | Structural modeling of ThsB (Thoeris TIR) from Bacillus amyloliquefaciens (Ba). (A) Center: Phyre2 modeling of BaThsB TIR-domain to the SARM1-TIR structure. Phyre2 model confidence: 96.2\%; BaThsB-TIR amino acid identity to SARM1-TIR: 15\%. Left: Alignment of SARM1-TIR (PDB ID: 6O0Q) to BaThsB-TIR. Right: Alignment of RPS4-TIR structure (PDB ID: 4C6R) to BaThsB-TIR (and to SARM1-TIR). (B) Phyre2 modeling of full length BaThsB to a putative signal transduction protein from Agathobacter rectales (a putative Thoeris system ThsB TIR). Phyre2 model confidence: 100\%; BaThsB amino acid identity to Agathobacter ThsB match: 59\%.

\section{PLANT TIR-DOMAIN SELF-ASSOCIATION IS NECESSARY FOR NADase ACTIVITY}

Plant TIR-TIR self-association occurs through at least two known interfaces formed by pairs of alpha helices (denoted as ' $\alpha$ ') (Bernoux et al., 2011; Williams et al., 2014, 2016). Both AE- (i.e., the $\alpha \mathrm{A} / \alpha \mathrm{E}$ surface) and DE-type ( $\alpha \mathrm{D} / \alpha \mathrm{E}$ surface) helical interfaces are necessary for TIR-TIR self-association, and, subsequent activation of the hypersensitive response. The DE interface was first revealed by the crystal structure of the flax L6 TIR domain (Bernoux et al., 2011). The RRS1 and RPS4 TIR heterodimer crystal indicated TIR-TIR contacts at the AE interfaces, while the RPP1 crystal revealed both $\mathrm{AE}$ and DE contacts (Williams et al., 2014; Zhang et al., 2017). Plant TIRdomains vary in strength of TIR-TIR self-associations and in some cases, self-association strength correlates with function (Schreiber et al., 2016; Zhang et al., 2017). The TIR-only protein, RBA1 (Response to HopBA1), self-associates using both AE and DE interfaces (Nishimura et al., 2017). RBA1 selfassociation is detectable via co-immunoprecipitation or yeast 2-hybrid assay, and both self-association interfaces must be intact to trigger cell death (Nishimura et al., 2017). Similarly, the isolated TIR-domain of the RPV1 TNL is sufficient to activate HR (Williams et al., 2016). However, self-association of RPV1 TIR-domains was not detectable by yeast two-hybrid analysis 


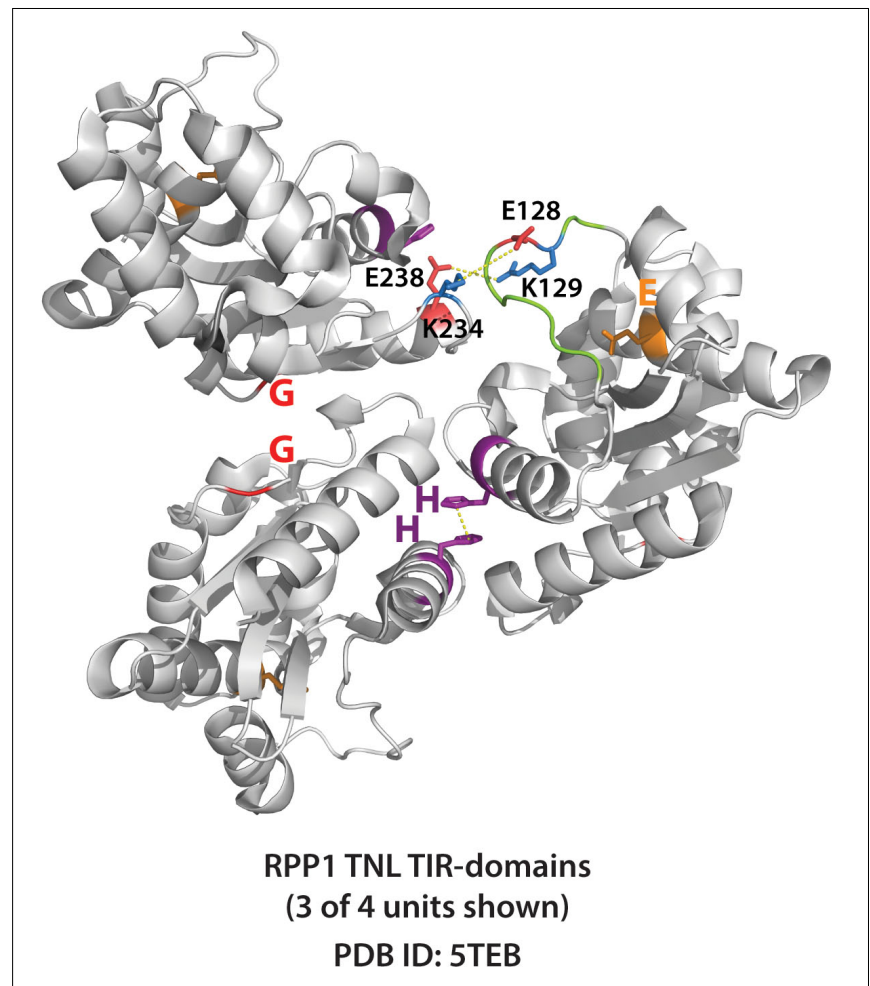

FIGURE 7 | Crystal structure of RPP1 plant TIR-domains showing TIR-TIR interfaces. Three of four RPP1 TIR-domain units shown (PDB ID: 5TEB). The AE (SH 108-109) and DE interfaces (G 229) are shown purple and red, respectively, while putative catalytic glutamate E164 shown orange. Connecting loop above putative catalytic glutamate is colored green. Potential loop interactions between putative ionic pairs of adjacent RPP1 monomers (residues E128 - K234, and R129 - E238) shown with dashed yellow lines. Distances between putative ionic pairs measured using Pymol: E128 to K234 (8.0 Å) and R129 to E238 (4.8 Å).

or size exclusion chromatography (Williams et al., 2016), yet disruption of the AE interface did abolish RPV1-mediated HR (Williams et al., 2016). Thus, intact TIR-TIR interfaces appear necessary for TIR-immune function, and can vary in strength. Additionally, the NBS-LRR domains of modular TNLs also promote oligomerization, and whether TIR-only proteins must evolve stronger TIR-TIR interfaces due to lack of NBS-LRR mediated organization is unclear.

Similar to cell death and disease resistance phenotypes, the activation of plant TIR NADase function requires both $\mathrm{AE}$ and DE self-association interfaces (Horsefield et al., 2019; Wan et al., 2019). It seems likely that the NADase activity of plant TIRs is dependent on some higher-order oligomer that has simultaneously engaged both $\mathrm{AE}$ and DE interfaces. Intriguingly, the RPP1 crystal structure (Figure 7) suggests that a loop that covers the catalytic glutamate could play this role, as it is positioned near a neighboring monomer only once both interfaces are engaged. Whether or not crystal structures of isolated TIR domains reflect the orientation in the activated TNL context remains to be determined. Currently, no structure of a full length TNL is available, and thus, how TNL oligomerization mediated by the NBS domains influences TIR-TIR associations, remains unclear. The activation of NADase activity following higher-order TIR oligomerization seems consistent with the behavior of the RBA1 E86A putative catalytic mutant (Wan et al., 2019). RBA1 E86A still self-associates (as measured by co-immunoprecipitation), suggesting that activation of $\mathrm{NAD}^{+}$hydrolysis follows the self-association of TIR-domains.

\section{OLIGOMERIC PLANT "RESISTOSOMES”}

The N-terminal coiled coil (CC) domain of some CC-domain type NLRs (e.g., Sr71, NRG1, MLA) can induce HR (Collier et al., 2011; Bai et al., 2012; Casey et al., 2016). Modeling of RPW8-type CC-domains suggests that they may adopt a 4helix bundle fold similar to that of the mixed-lineage kinase-like protein family of animals, which insert into host membranes and promote cell death (Jubic et al., 2019). Recently, cryo-EM structures for active (ATP-bound) and inactive (ADP-bound) ZAR1 'resistosomes' were determined (Wang et al., 2019a,b). The ZAR1 (HOPZ-ACTIVATED RESISTANCE 1) resistosome complex forms a ring-shaped pentameric structure, and contains bound RKS1 pseudokinase, and an effector-modified kinase, PBL2. The pentameric resistosome structure is driven by the ZAR1 NBS-LRR domains, however, the presence of associated host guardee and adaptor proteins (e.g., RKS1, PBL2) will also influence overall resistosome structure (Wang et al., 2019a). The N-terminal CC-domains of ZAR1 subunits undergo a conformational change, each extending a helix to form a funnellike structure, which is hypothesized to disrupt membrane integrity and promote cell death (Wang et al., 2019a).

Can the pentameric structure of the ZAR1 resistosome - a CC-domain type NLR - inform what higher order complexes an activated TNL might form? It is enticing to speculate that, like ZAR1 and animal NLRs, an oligomeric TNL NADase complex also forms a ring-shaped resistosome? A variety of stoichiometries are observed for the animal NLR oligomers that form the apoptosome and inflammosome rings (Zhang et al., 2015). The hypothetical TNL resistosome could be of a range of stoichiometries, and most likely forms a ring. However, given the existing structures of plant TIR domains, it seems difficult to reconcile the radial (head to tail) symmetry of a ring-shaped resistosome, no matter the stoichiometry. In these structures, the $\mathrm{AE}$ and DE interfaces are in a "head to head" orientation that seems at odds with a circular chain. Perhaps an increase in local concentration of TIR domains is sufficient to promote signaling. Or possibly, these interfaces will not be seen in the context of a full-length TNL oligomer structure. Fusion of the SARM1 SAM domains to either the N-terminus (Horsefield et al., 2019; Wan et al., 2019) or C-terminus (unpublished) of plant TIR-domains enables NADase activity and HR-induction. The SAM domains of SARM1 form an octameric ring (Figure 5B) (Horsefield et al., 2019; Sporny et al., 2019). Even in the context of a fusion protein with forced oligomerization, the RPS4 SAM:TIR protein still requires both $\mathrm{AE}$ and $\mathrm{DE}$ interfaces (Wan et al., 2019). These results suggest that an octameric ring structure can accommodate plant TIR function, and also that there is surprising flexibility in how functional TIR domain oligomerization can be promoted. 


\section{ZAR1 Resistosome (PDB ID: 6J5T)}
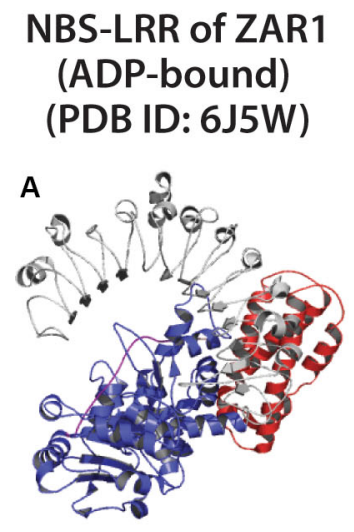

NBS-LRR of ZAR1 (ATP-bound) (PDB ID: 6J5T)

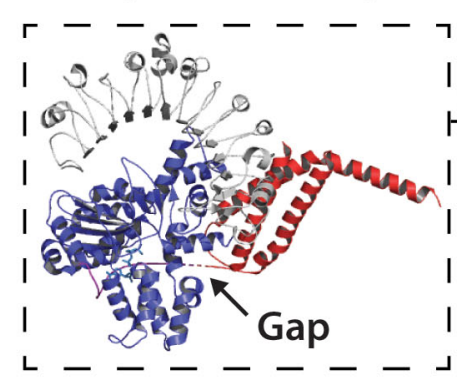

B

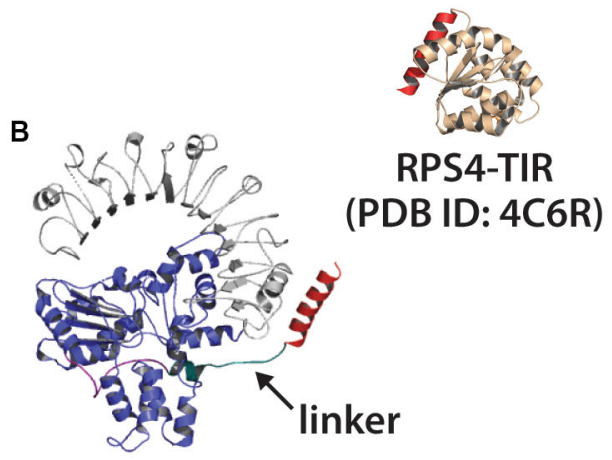

RPS4 NBS-LRR modeled to 6J5T

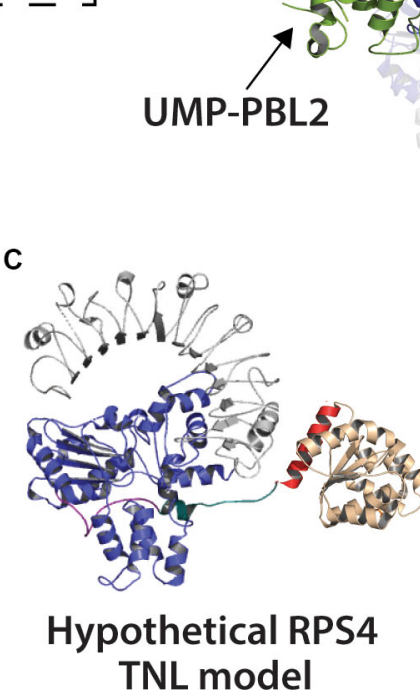

FIGURE 8 | Modeling of the RPS4 NBS-LRR (a TNL) to the NBS-LRR of ZAR1 resistosome (ATP-bound) or ZAR1 monomer (ADP-bound). (A) Left: ADP-bound ZAR1 monomer structure as determined by Wang et al. (2019a). Center: single ATP-bound ZAR1 (CNL) subunit from the cryo-EM determined resistosome structure by Wang et al. (2019b). Right: Activated ZAR1-resistosome. Coiled coil (CC) domain of ZAR1 colored red. NBS (nucleotide binding site) colored blue and LRR (leucine rich repeat) colored gray. ZAR1 N-terminal linker regions colored purple, and gaps in linker indicated by arrow. Resistosome-associated proteins RKS1 and effector-modified UMP-PBL2 shown tan and green, respectively. (B) Left: Phyre2 modeling of the RPS4 NBS-LRR (including final helix of RPS4 TIR-domain shown in red) to ATP-bound NBS-LRR of the ZAR1 resistosome (PDB ID: 6J5T). The putative RPS4 linker is colored teal and indicated with arrow. Above and right: crystal structure of RPS4 (TNL) TIR-domain (PDB ID: 4C6R) with putative catalytic glutamate (E88) colored orange. (C) The RPS4 TIR manually docked onto the RPS4 NBS-LRR model. The red helix shown on RPS4-TIR is the same red helix included in the NBS-LRR model.

Using Phyre2, we modeled the NBS-LRR domains of RPS4 onto the structures of inactive and active ZAR1 NBS-LRRs (Figure 8). The NBS and N-terminal linker regions of RPS4, as compared with ZAR1, are similar in length and potentially in orientation (Figure 8). While entirely speculative, there would appear to be limits on the amount of rotational flexibility the TIR domains would have in a hypothetical resistosome to engage in simultaneous $\mathrm{AE}$ and $\mathrm{DE}$ interfaces. The oligomerization state of so-called "paired NLRs" - where individual partners typically assume a 'sensor' or 'signal' role - may be even more complex. Given that RPS4 and RRS1 appear to function in a complex (Huh et al., 2017), what would the stoichiometry and organization of a hetero-oligomeric resistosome look like? The fact that the
RRS1 TIR lacks a catalytic glutamate makes the situation even more interesting.

Plant TIR-only proteins can signal despite their lack of C-terminal NBS-LRR domains (Nishimura et al., 2017; Wan et al., 2019). In the absence of oligomerizing NBS-LRR domains, what higher order structures might naturally occurring TIRonly proteins form? The TIR-only protein, RBA1, self-associates and requires the conserved $\mathrm{AE}$ and DE-type interfaces. Are TIR-only oligomers different than TNL oligomers? RBA1 also requires EDS1 and NRG1, but like TNL receptors there is still no clear mechanistic link between TIR activation and downstream signal transduction (Nishimura et al., 2017; Wan et al., 2019). 


\section{HOW MIGHT PLANT TIR-NADases TRANSMIT IMMUNE SIGNALS?}

$\mathrm{NAD}^{+}$is a major cellular metabolite, redox carrier, and substrate for numerous processes including DNA repair, epigenetic modifications, immunity and signaling (Adams-Phillips et al., 2010; Petriacq et al., 2013; Petriacq et al., 2016). Activated plant TIR-domains are $\mathrm{NAD}^{+}$-hydrolases, but how might $\mathrm{NAD}^{+}$. consumption activate immune responses? SARM1 apparently triggers cell death by depleting $\mathrm{NAD}^{+}$, but plant TIRs do not cause detectable $\mathrm{NAD}^{+}$reductions in planta (Wan et al., 2019). One possibility is that $\mathrm{NAD}^{+}$consumption by plant TIRs generates signal molecules that turn on downstream immune components.

Unlike SARM1, plant TIRs did not generate c-ADPR, but instead produced $\mathrm{v}$-cADPR, both in vitro and after transient expression in N. benthamiana (Wan et al., 2019). Moreover, $\mathrm{v}$-cADPR was also produced by activation of RBA1 after bacterial delivery of the Pseudomonas syringae effector HopBA1 (Wan et al., 2019). Neither EDS1 or NRG1 - downstream TIR-signaling components - were required for $\mathrm{v}$-cADPR generation by activated TIRs in planta (Wan et al., 2019). These results indicate that $\mathrm{v}$-cADPR accumulation is upstream of both cell death and the known signaling components downstream of TIR proteins. Curiously, the in planta generation of v-cADPR by TIR-domains isolated from TNLs was nearly 100 -fold lower than that of TIRonly proteins (Wan et al., 2019). Is this difference an artifact of truncating TNL proteins, or an intrinsic difference between TIR-only and TNL TIR-domains? Whether an auto-active variant of a full length TNL might produce comparable v-cADPR to TIR-only proteins has not been examined. It is also unclear if the context of a full length NLR could influence the ratio or type of products generated by $\mathrm{NAD}^{+}$-hydrolysis, apart from hydrolysis kinetics.

The v-cADPR molecule appears to uniquely identify plant TIR-driven ETI, as MLA10 expression and RPM1 activation (both CNLs) did not elevate v-cADPR (Wan et al., 2019). The chemical structure of v-cADPR is presently unknown, and could vary significantly from cyclic-ADPR. It is possible that v-cADPR shares signaling properties with other $\mathrm{NAD}^{+}$-derivatives such as cyclic-ADPR, ADPR, and NAAD (a product of the SARM1-TIR), which are potent $\mathrm{Ca}^{2+}$ channel activators (Lee, 2012; Guse, 2015). Numerous studies reveal $\mathrm{Ca}^{2+}$ signaling is necessary for plant immunity and HR-driven cell death (Grant et al., 2000; Ma and Berkowitz, 2007, 2011; Marcec et al., 2019). Intriguingly, cyclicADPR has been reported to trigger plant defense gene expression, and a calcium channel blocker, lanthanum chloride, prevents plant cell death and HR (although this is not specific to TIR phenotypes) (Durner et al., 1998; Grant et al., 2000).

At this point v-cADPR can be considered a biomarker for plant TIR activity, as its production is correlated with TIR function, however, it is not clear if it is either necessary or sufficient to trigger cell death or disease resistance. In vitro assays indicate that the TIR-only proteins RBA1 and BdTIR are also capable of cleaving $\mathrm{NADP}^{+}$(Wan et al., 2019), and it remains to be determined what the putative $\mathrm{v}$-cADPRP product looks like and if it is produced in planta. Are there other, as yet, unidentified products? How NADaseproduced signaling products might activate immune responses is entirely speculative, but a reasonable candidate to receive a signal would be EDS1, potentially mediated by an EDS1 hetero-oligomer surface. The fact that EDS1/SAG101 and EDS1/PAD4 heterodimers can have non-redundant functions, with specificity in regards to the particular activating TIR (Cui et al., 2017; Castel et al., 2019; Lapin et al., 2019; Wu et al., 2019), complicate simple models where TIR proteins generate a generic signal.

Because $\mathrm{NAD}^{+}$levels influence numerous cellular processes, the consumption of $\mathrm{NAD}^{+}$by plant TIRs during immunity could impact myriad cellular responses. For instance, extracellular $\mathrm{NAD}^{+}\left(\mathrm{eNAD}^{+}\right)$is a potent immunostimulatory signal and reducing $\mathrm{NAD}^{+}$levels compromises disease resistance; conversely, eNAD ${ }^{+}$application can bolster immunity (Zhang and Mou, 2012; Wang et al., 2016; Mou, 2017; Alferez et al., 2018). Likewise, the AvrRxol and RipN, virulence-promoting effectors of plant pathogens, can modulate host $\mathrm{NAD}^{+}$homeostasis and defense responses (Schuebel et al., 2016; Shidore et al., 2017; Sun et al., 2019). While total $\mathrm{NAD}^{+}$levels did not obviously change with TIR expression (Wan et al., 2019), it's possible that localization of NADase activity could have an impact on output.

\section{TIR-PROTEINS ACROSS PLANT PHYLOGENIES}

TIR-domain encoding genes can be found in almost all plant lineages. However, the class and abundance of encoded TIRproteins can vary widely between species (Collier et al., 2011; Yue et al., 2012; Nandety et al., 2013; Sun et al., 2014; Gao et al., 2018). Particularly, between dicot and monocot plant species, the complement of CNL vs. TNL-type NLRs can vary greatly (Sun et al., 2014; Gao et al., 2018). Canonical TNLtype resistance genes are absent from all examined monocot genomes, as are the TIR-pathway mediators, SAG101 and NRG1 (Collier et al., 2011; Wagner et al., 2013). Remarkably, convergent loss of TNLs and downstream genes has occurred several times during plant evolution (Collier et al., 2011; Baggs et al., 2019). Monocots do, however, encode several TIR-NBS and TIR-only genes, although in low abundance relative to the high number of TNLs commonly present in dicots (Sun et al., 2014; Gao et al., 2018). Whether or not these monocot TIR proteins are functioning as immune receptors remains to be determined. However, the TIR-only protein RBA1, can trigger cell death in response a specific pathogen effector, and both TIR-NBS and TIR-X proteins from various plant species are reported to enhance immunity (Meyers et al., 2002; Staal et al., 2008; Nandety et al., 2013; Zhao et al., 2015; Nishimura et al., 2017; Chen et al., 2018; Santamaria et al., 2019). Thus, while TNLs may be absent from monocot genomes, TIR-signaling could play roles in regulating physiological responses and immunity in monocots. BdTIR, a TIR-only protein from the monocot Brachypodium, has many of the hallmarks of dicot TIR domains: it has the conserved putative catalytic glutamic acid, produces v-cADPR and triggers EDS1-dependent cell death in N. benthamiana (Wan 
et al., 2019). Intriguingly, BdTIR cell death in N. benthamiana is also dependent on the downstream TIR signaling component NbNRG1, despite the fact that monocots have lost NRG1 from their genomes. Therefore, it is possible that TIR-domains from distant plant phylogenies produce common signals from $\mathrm{NAD}^{+}$-hydrolysis, while the putative immune output depends on which downstream components (e.g., EDS1-members, NRG1) are present to enact the signal.

While TNLs are absent from monocots (and several dicot lineages), they are present broadly across the plant phylogeny, including bryophytes and conifers (see also Figure 2C) (Baggs et al., 2019). For instance, the moss Physcomitrella patens carries TNL loci, as does the western white pine, Pinus monticola (Liu and Ekramoddoullah, 2011; Tanigaki et al., 2014). Two pine TNL loci, TNL1 and TNL2, are correlated with blister rust resistance (Liu and Ekramoddoullah, 2011). TIR-domainencoding genes were more recently reported in the agriculturally important red algae, Pyropia yezoensis, which is used for nori production (Tang et al., 2019). At least one TIR-domain encoding gene, along with several NBS genes of Pyropia are upregulated by challenge with the oomycete pathogen, Pythium (Tang et al., 2019). Genes with TIR immune receptor-like domain combinations have been found in the genomes of green algae. Botryococcus contains TIR-NBS encoding genes, while remarkably, Chromochloris has NLR-like genes that contain all three canonical NLR domains (TIR, NBS and LRR) (Shao et al., 2019). More functional evidence for algal TIRs or TNLs in immunity is needed, as well as investigation into the algal relatives of downstream TIR pathway components defined in dicots. It seems likely that TIR-domains across photosynthetic organisms harbor NADase activities, however, this has not been explored. Nor is it clear if these TIR-domains could produce similar molecules from $\mathrm{NAD}^{+}$-hydrolysis. An expanded collection of genomic data from algae and early plant clades will help to assess both the conservation and abundance of putative TIRimmune pathways.

\section{(MORE) UNANSWERED QUESTIONS?}

TIR-domains encoded by species from all domains of life are now known to play roles in immunity. Recent studies now suggest a new paradigm of TIR-mediated immunity in plants: the oligomerization and self-association of TIR-domains, and subsequent hydrolysis of $\mathrm{NAD}^{+}$(Zhang et al., 2017; Horsefield et al., 2019; Wan et al., 2019). Many important and intriguing questions about TIR-immunity remain. For instance, the stoichiometry and confirmation of active plant TNL or TIRimmune complexes is not known. Furthermore, does the NADase activity of plant TIRs generate immunomodulatory signals? And if so, how are these signals transduced and decoded? Finally, the extent of plant TIR functional conservation is not fully known; i.e., are the TIR-domains encoded by more distantly-related photosynthetic lineages also NADases and do they function in or outside of immunity?

If plant TIRs generate immunomodulatory signals from the hydrolysis of $\mathrm{NAD}(\mathrm{P})^{+}$, then what is that signal? For instance, might variant-cADPR per se be sufficient to activate transcriptional defenses, or the hypersensitivity response? Or might different TIR-derived signal molecules communicate different outputs? Additionally, plant TIR-NADases could potentially regulate $\mathrm{NAD}^{+}$levels and cellular metabolism apart from immune signal generation. Do TIR-domains from all plant lineages generate the same type(s) of signals, and how has evolution shaped the components which sense and translate outputs from these signals? The subcellular localization and expression of both signal generating TIRs, and downstream signal receivers could influence potential response outcomes.

TIR-based immunity appears to have an ancient role in prokaryotes as an anti-viral defense system (Doron et al., 2018; Cohen et al., 2019). The conservation of NADase activity among animal, plant and prokaryotic TIRs suggests that an ancient enzymatic activity has been re-purposed multiple times in eukaryotic evolution to promote cell death or immune function. A particularly intriguing question is how did plant TNLs and TIRs evolve to become reliant on the downstream EDS1family and 'helper' NLR partners? Presumably, these components independently provided host benefits, prior to co-evolution into overlapping networks. An in-depth analysis of genomes from early plant lineages may provide insights into how TIRs, EDS1members and 'helper' NLRs co-evolved to function in a core pathway, and provide clues into the mechanisms of TIR-signaling networks of higher plants.

Combined biochemical and evolutionary approaches may provide guidance into how variation in the TIR active site or TIR association interfaces could affect immune outputs. In the future, such findings may be able to offer predictions regarding the kinetic properties of specific TIR-domains, as well as a likely profile of $\mathrm{NAD}^{+}$-derived products. For instance, might modulating $\mathrm{NAD}^{+}$-hydrolysis kinetics and/or product profile influence the type or strength of immune output? Can in vitro evolution enable 'tweaking' of TIR-active sites, or of TIR-TIR self-association interfaces, and thus alter the profile of products derived from $\mathrm{NAD}^{+}$-hydrolysis?

The recognition that TIR domains across the tree of life have conserved enzymatic functions has opened new avenues of investigation into the plant immune system. While much remains undiscovered, the field is poised to describe fully connected NLR signaling pathways that lead to immune outputs. This synthesis will enable rational engineering of plant immunity to help address the increasing demands on our agricultural systems.

\section{AUTHOR CONTRIBUTIONS}

$\mathrm{AB}$ and $\mathrm{MN}$ wrote the manuscript. Both authors approved the manuscript.

\section{FUNDING}

This work was supported by the National Science Foundation (IOS-1758400) and startup funds from Colorado State University to $\mathrm{MN}$. 


\section{REFERENCES}

Adams-Phillips, L., Briggs, A. G., and Bent, A. F. (2010). Disruption of poly(ADPribosyl)ation mechanisms alters responses of Arabidopsis to biotic stress. Plant Physiol. 152, 267-280. doi: 10.1104/pp.109.148049

Alaidarous, M., Ve, T., Casey, L. W., Valkov, E., Ericsson, D. J., Ullah, M. O., et al. (2014). Mechanism of bacterial interference with TLR4 signaling by Brucella Toll/interleukin-1 receptor domain-containing protein TcpB. J. Biol. Chem. 289, 654-668. doi: 10.1074/jbc.M113.523274

Alferez, F. M., Gerberich, K. M., Li, J. L., Zhang, Y., Graham, J. H., and Mou, Z. (2018). Exogenous nicotinamide adenine dinucleotide induces resistance to citrus canker in citrus. Front. Plant Sci. 9:1472. doi: 10.3389/fpls.2018.01472

Baggs, E., Thanki, A., O'Grady, R., Schudoma, C., Haerty, W., and Krasileva, K. (2019). Convergent loss of an EDS1/PAD4 signalling pathway in several plant lineages predicts new components of plant immunity and drought response. bioRxiv [preprint]. doi: 10.1101/572560

Bai, S., Liu, J., Chang, C., Zhang, L., Maekawa, T., Wang, Q., et al. (2012). Structure-function analysis of barley NLR immune receptor MLA10 reveals its cell compartment specific activity in cell death and disease resistance. PLoS Pathog 8:e1002752. doi: 10.1371/journal.ppat.1002752

Bernoux, M., Ve, T., Williams, S., Warren, C., Hatters, D., Valkov, E., et al. (2011). Structural and functional analysis of a plant resistance protein TIR domain reveals interfaces for self-association, signaling, and autoregulation. Cell Host Microbe 9, 200-211. doi: 10.1016/j.chom.2011.02.009

Bhandari, D. D., Lapin, D., Kracher, B., von Born, P., Bautor, J., Niefind, K., et al. (2019). An EDS1 heterodimer signalling surface enforces timely reprogramming of immunity genes in Arabidopsis. Nat. Commun. 10, 772. doi: 10.1038/s41467-019-08783-0

Boutrot, F., and Zipfel, C. (2017). Function, discovery, and exploitation of plant pattern recognition receptors for broad-spectrum disease resistance. Annu. Rev. Phytopathol. 55, 257-286. doi: 10.1146/annurev-phyto-080614- 120106

Bovijn, C., Ulrichts, P., De Smet, A. S., Catteeuw, D., Beyaert, R., Tavernier, J., et al. (2012). Identification of interaction sites for dimerization and adapter recruitment in Toll/interleukin-1 receptor (TIR) domain of Toll-like receptor 4. J. Biol. Chem. 287, 4088-4098. doi: 10.1074/jbc.M111.282350

Casey, L. W., Lavrencic, P., Bentham, A. R., Cesari, S., Ericsson, D. J., Croll, T., et al. (2016). The CC domain structure from the wheat stem rust resistance protein Sr33 challenges paradigms for dimerization in plant NLR proteins. Proc. Natl. Acad. Sci. U.S.A. 113, 12856-12861. doi: 10.1073/pnas.1609922113

Castel, B., Ngou, P. M., Cevik, V., Redkar, A., Kim, D. S., Yang, Y., et al. (2019). Diverse NLR immune receptors activate defence via the RPW8-NLR NRG1. New Phytol. 222, 966-980. doi: 10.1111/nph.15659

Chan, S. L., Low, L. Y., Hsu, S., Li, S., Liu, T., Santelli, E., et al. (2009). Molecular mimicry in innate immunity: crystal structure of a bacterial TIR domain. J. Biol. Chem. 284, 21386-21392. doi: 10.1074/jbc.C109.007591

Chen, G., Wei, B., Li, G., Gong, C., Fan, R., and Zhang, X. (2018). TaEDS1 genes positively regulate resistance to powdery mildew in wheat. Plant Mol. Biol. 96, 607-625. doi: 10.1007/s11103-018-0718-9

Cohen, D., Melamed, S., Millman, A., Shulman, G., Oppenheimer-Shaanan, Y., Kacen, A., et al. (2019). Cyclic GMP-AMP signalling protects bacteria against viral infection. Nature 574, 691-695. doi: 10.1038/s41586-019-1605-5

Collier, S. M., Hamel, L. P., and Moffett, P. (2011). Cell death mediated by the $\mathrm{N}$-terminal domains of a unique and highly conserved class of NB-LRR protein. Mol. Plant Microbe Interact. 24, 918-931. doi: 10.1094/MPMI-03-11-0050

Coronas-Serna, J. M., Louche, A., Rodríguez-Escudero, M., Roussin, M., Imbert, P. R. C., Rodríguez-Escudero, I., et al. (2019). The TIR-domain containing effectors BtpA and BtpB from Brucella abortus block energy metabolism. bioRxiv [Preprint]. doi: 10.1101/703330

Cui, H., Gobbato, E., Kracher, B., Qiu, J., Bautor, J., and Parker, J. E. (2017). A core function of EDS1 with PAD4 is to protect the salicylic acid defense sector in Arabidopsis immunity. New Phytol. 213, 1802-1817. doi: 10.1111/nph.14302

Dangl, J. L., Horvath, D. M., and Staskawicz, B. J. (2013). Pivoting the plant immune system from dissection to deployment. Science 341, 746-751. doi: $10.1126 /$ science. 1236011

Doron, S., Melamed, S., Ofir, G., Leavitt, A., Lopatina, A., Keren, M., et al. (2018). Systematic discovery of antiphage defense systems in the microbial pangenome. Science 359:eaar4120. doi: 10.1126/science.aar4120
Durner, J., Wendehenne, D., and Klessig, D. F. (1998). Defense gene induction in tobacco by nitric oxide, cyclic GMP, and cyclic ADP-ribose. Proc. Natl. Acad. Sci. U.S.A. 95, 10328-10333. doi: 10.1073/pnas.95.17.10328

Essuman, K., Summers, D. W., Sasaki, Y., Mao, X., DiAntonio, A., and Milbrandt, J. (2017). The SARM1 Toll/Interleukin-1 receptor domain possesses intrinsic $\mathrm{NAD}(+)$ cleavage activity that promotes pathological axonal degeneration. Neuron 93, 1334-1343.e5. doi: 10.1016/j.neuron.2017.02.022

Essuman, K., Summers, D. W., Sasaki, Y., Mao, X., Yim, A. K. Y., DiAntonio, A., et al. (2018). TIR domain proteins are an ancient family of $\mathrm{NAD}(+)$-consuming enzymes. Curr. Biol. 28, 421430.e4. doi: 10.1016/j.cub.2017.12.024

Feys, B. J., Wiermer, M., Bhat, R. A., Moisan, L. J., Medina-Escobar, N., Neu, C., et al. (2005). Arabidopsis SENESCENCE-ASSOCIATED GENE101 stabilizes and signals within an ENHANCED DISEASE SUSCEPTIBILITY1 complex in plant innate immunity. Plant Cell. 17, 2601-2613. doi: 10.1105/tpc.105.033910

Gao, Y., Wang, W., Zhang, T., Gong, Z., Zhao, H., and Han, G. Z. (2018). Out of water: the origin and early diversification of plant R-Genes. Plant Physiol. 177, 82-89. doi: 10.1104/pp.18.00185

Gerdts, J., Brace, E. J., Sasaki, Y., DiAntonio, A., and Milbrandt, J. (2015). SARM1 activation triggers axon degeneration locally via $\mathrm{NAD}(+)$ destruction. Science 348, 453-457. doi: 10.1126/science. 1258366

Grant, M., Brown, I., Adams, S., Knight, M., Ainslie, A., and Mansfield, J. (2000). The RPM1 plant disease resistance gene facilitates a rapid and sustained increase in cytosolic calcium that is necessary for the oxidative burst and hypersensitive cell death. Plant J. 23, 441-450. doi: 10.1046/j.1365-313x.2000. 00804.x

Guse, A. H. (2015). Calcium mobilizing second messengers derived from NAD. Biochim. Biophys. Acta 1854, 1132-1137. doi: 10.1016/j.bbapap.2014.12.015

Heidrich, K., Wirthmueller, L., Tasset, C., Pouzet, C., Deslandes, L., and Parker, J. E. (2011). Arabidopsis EDS1 connects pathogen effector recognition to cell compartment-specific immune responses. Science 334, 1401-1404. doi: 10. 1126/science. 1211641

Horsefield, S., Burdett, H., Zhang, X., Manik, M. K., Shi, Y., Chen, J., et al. (2019). $\mathrm{NAD}(+)$ cleavage activity by animal and plant TIR domains in cell death pathways. Science 365, 793-799. doi: 10.1126/science.aax1911

Hu, Z., Zhou, Q., Zhang, C., Fan, S., Cheng, W., Zhao, Y., et al. (2015). Structural and biochemical basis for induced self-propagation of NLRC4. Science 350, 399-404. doi: 10.1126/science.aac5489

Huh, S. U., Cevik, V., Ding, P., Duxbury, Z., Ma, Y., Tomlinson, L., et al. (2017). Protein-protein interactions in the RPS4/RRS1 immune receptor complex. PLoS Pathog 13:e1006376. doi: 10.1371/journal.ppat.1006376

Jones, J. D., and Dangl, J. L. (2006). The plant immune system. Nature 444, 323-329.

Jubic, L. M., Saile, S., Furzer, O. J., El Kasmi, F., and Dangl, J. L. (2019). Help wanted: helper NLRs and plant immune responses. Curr. Opin. Plant Biol. 50, 82-94. doi: 10.1016/j.pbi.2019.03.013

Kenny, E. F., and O'Neill, L. A. (2008). Signalling adaptors used by Toll-like receptors: an update. Cytokine 43, 342-349. doi: 10.1016/j.cyto.2008.07.010

Lapin, D., Kovacova, V., Sun, X., Dongus, J. A., Bhandari, D. D., von Born, P., et al. (2019). A coevolved EDS1-SAG101-NRG1 module mediates cell death signaling by TIR-domain immune receptors. Plant Cell 31, 24302455, doi: 10.1105/tpc.19.00118

Lee, H. C. (2012). Cyclic ADP-ribose and nicotinic acid adenine dinucleotide phosphate (NAADP) as messengers for calcium mobilization. J. Biol. Chem. 287, 31633-31640. doi: 10.1074/jbc.R112.349464

Lee, H. C., and Zhao, Y. J. (2019). Resolving the topological enigma in $\mathrm{Ca}(2+)$ signaling by cyclic ADP-ribose and NAADP. J. Biol. Chem. 294, 19831-19843. doi: 10.1074/jbc.REV119.009635

Liu, J. J., and Ekramoddoullah, A. K. (2011). Genomic organization, induced expression and promoter activity of a resistance gene analog (PmTNL1) in western white pine (Pinus monticola). Planta 233, 1041-1053. doi: 10.1007/ s00425-011-1353-8

Loring, H. S., Icso, J. D., Nemmara, V., and Thompson, P. R. (2020). Initial kinetic characterization of sterile alpha and Toll/Interleukin receptor motif-containing protein 1. Biochemistry 59, 933942. doi: 10.1021/acs.biochem.9b01078

Ma, W., and Berkowitz, G. A. (2007). The grateful dead: calcium and cell death in plant innate immunity. Cell Microbiol. 9, 2571-2585. doi: 10.1111/j.1462-5822. 2007.01031.x 
Ma, W., and Berkowitz, G. A. (2011). Ca2+ conduction by plant cyclic nucleotide gated channels and associated signaling components in pathogen defense signal transduction cascades. New Phytol. 190, 566-572. doi: 10.1111/j.1469-8137. 2010.03577.x

Marcec, M. J., Gilroy, S., Poovaiah, B. W., and Tanaka, K. (2019). Mutual interplay of $\mathrm{Ca}(2+)$ and ROS signaling in plant immune response. Plant Sci. 283, 343-354. doi: $10.1016 /$ j.plantsci.2019.03.004

Meyers, B. C., Morgante, M., and Michelmore, R. W. (2002). TIR-X and TIRNBS proteins: two new families related to disease resistance TIR-NBS-LRR proteins encoded in Arabidopsis and other plant genomes. Plant J. 32, 77-92. doi: 10.1046/j.1365-313x.2002.01404.x

Mou, Z. (2017). Extracellular pyridine nucleotides as immune elicitors in arabidopsis. Plant Signal. Behav. 12:e1388977. doi: 10.1080/15592324.2017. 1388977

Murata, H., Khine, C. C., Nishikawa, A., Yamamoto, K. I., Kinoshita, R., and Sakaguchi, M. (2018). c-Jun N-terminal kinase (JNK)-mediated phosphorylation of SARM1 regulates $\mathrm{NAD}(+)$ cleavage activity to inhibit mitochondrial respiration. J. Biol. Chem. 293, 18933-18943. doi: 10.1074/jbc. RA118.004578

Nandety, R. S., Caplan, J. L., Cavanaugh, K., Perroud, B., Wroblewski, T., Michelmore, R. W., et al. (2013). The role of TIR-NBS and TIR-X proteins in plant basal defense responses. Plant Physiol. 162, 1459-1472. doi: 10.1104/pp. 113.219162

Nanson, J. D., Kobe, B., and Ve, T. (2019). Death, TIR, and RHIM: self-assembling domains involved in innate immunity and cell-death signaling. J. Leukoc. Biol. 105, 363-375. doi: 10.1002/JLB.MR0318-123R

Nimma, S., Ve, T., Williams, S. J., and Kobe, B. (2017). Towards the structure of the TIR-domain signalosome. Curr. Opin. Struct. Biol. 43, 122-130. doi: 10.1016/j.sbi.2016.12.014

Nishimura, M. T., Anderson, R. G., Cherkis, K. A., Law, T. F., Liu, Q. L., Machius, M., et al. (2017). TIR-only protein RBAl recognizes a pathogen effector to regulate cell death in Arabidopsis. Proc. Natl. Acad. Sci. U.S.A. 114, E2053E2062. doi: 10.1073/pnas.1620973114

Nyman, T., Stenmark, P., Flodin, S., Johansson, I., Hammarstrom, M., and Nordlund, P. (2008). The crystal structure of the human tolllike receptor 10 cytoplasmic domain reveals a putative signaling dimer. J. Biol. Chem. 283, 11861-11865. doi: 10.1074/jbc.C8000 01200

O'Neill, L. A., and Bowie, A. G. (2007). The family of five: TIR-domain-containing adaptors in Toll-like receptor signalling. Nat. Rev. Immunol. 7, 353-364. doi: 10.1038/nri2079

Peart, J. R., Mestre, P., Lu, R., Malcuit, I., and Baulcombe, D. C. (2005). NRG1, a CC-NB-LRR protein, together with $\mathrm{N}$, a TIR-NB-LRR protein, mediates resistance against tobacco mosaic virus. Curr. Biol. 15, 968-973. doi: 10.1016/j. cub.2005.04.053

Petriacq, P., de Bont, L., Tcherkez, G., and Gakiere, B. (2013). NAD: not just a pawn on the board of plant-pathogen interactions. Plant Signal. Behav. 8:e22477. doi: $10.4161 /$ psb.22477

Petriacq, P., Ton, J., Patrit, O., Tcherkez, G., and Gakiere, B. (2016). NAD Acts as an Integral Regulator of Multiple Defense Layers. Plant Physiol. 172, 1465-1479. doi: $10.1104 / \mathrm{pp} .16 .00780$

Qi, D., and Innes, R. W. (2013). Recent advances in plant NLR structure, function, localization, and signaling. Front. Immunol. 4:348. doi: 10.3389/fimmu.2013. 00348

Qi, T., Seong, K., Thomazella, D. P. T., Kim, J. R., Pham, J., Seo, E., et al. (2018). NRG1 functions downstream of EDS1 to regulate TIR-NLR-mediated plant immunity in Nicotiana benthamiana. Proc. Natl. Acad. Sci. U.S.A. 115, E10979-E10987. doi: 10.1073/pnas.1814856115

Santamaria, M. E., Martinez, M., Arnaiz, A., Rioja, C., Burow, M., Grbic, V., et al. (2019). An Arabidopsis TIR-Lectin two-domain protein confers defense properties against Tetranychus urticae. Plant Physiol. 179, 1298-1314. doi: 10.1104/pp.18.00951

Schreiber, K. J., Bentham, A., Williams, S. J., Kobe, B., and Staskawicz, B. J. (2016). Multiple domain associations within the arabidopsis immune receptor RPP1 regulate the activation of programmed cell death. PLoS Pathog 12:e1005769. doi: 10.1371/journal.ppat.100576

Schuebel, F., Rocker, A., Edelmann, D., Schessner, J., Brieke, C., and Meinhart, A. (2016). 3'-NADP and 3'-NAADP, Two Metabolites Formed by the Bacterial
Type III Effector AvrRxo1. J. Biol. Chem. 291, 22868-22880. doi: 10.1074/jbc. M116.751297

Shao, Z. Q., Xue, J. Y., Wang, Q., Wang, B., and Chen, J. Q. (2019). Revisiting the Origin of Plant NBS-LRR Genes. Trends Plant Sci. 24, 9-12. doi: 10.1016/j. tplants.2018.10.015

Shidore, T., Broeckling, C. D., Kirkwood, J. S., Long, J. J., Miao, J., Zhao, B., et al. (2017). The effector AvrRxol phosphorylates NAD in planta. PLoS Pathog 13:e1006442. doi: 10.1371/journal.ppat.1006442

Shivers, R. P., Kooistra, T., Chu, S. W., Pagano, D. J., and Kim, D. H. (2009). Tissue-specific activities of an immune signaling module regulate physiological responses to pathogenic and nutritional bacteria in C. Elegans. Cell Host Microbe 6, 321-330. doi: 10.1016/j.chom.2009.09.001

Snyder, G. A., Deredge, D., Waldhuber, A., Fresquez, T., Wilkins, D. Z., Smith, P. T., et al. (2014). Crystal structures of the Toll/Interleukin-1 receptor (TIR) domains from the Brucella protein TcpB and host adaptor TIRAP reveal mechanisms of molecular mimicry. J. Biol. Chem. 289, 669-679. doi: 10.1074/jbc.M113.523407

Spear, A. M., Loman, N. J., Atkins, H. S., and Pallen, M. J. (2009). Microbial TIR domains: not necessarily agents of subversion? Trends Microbiol. 17, 393-398. doi: 10.1016/j.tim.2009.06.005

Sporny, M., Guez-Haddad, J., Lebendiker, M., Ulisse, V., Volf, A., Mim, C., et al. (2019). Structural evidence for an octameric ring arrangement of SARM1. J. Mol. Biol. 431, 3591-3605. doi: 10.1016/j.jmb.2019.06.030

Staal, J., Kaliff, M., Dewaele, E., Persson, M., and Dixelius, C. (2008). RLM3, a TIR domain encoding gene involved in broad-range immunity of Arabidopsis to necrotrophic fungal pathogens. Plant J. 55, 188-200. doi: 10.1111/j.1365-313X. 2008.03503.x

Sun, X., Pang, H., Li, M., Chen, J., and Hang, Y. (2014). Tracing the origin and evolution of plant TIR-encoding genes. Gene 546, 408-416. doi: 10.1016/j.gene. 2014.04.060

Sun, Y., Li, P., Shen, D., Wei, Q., He, J., and Lu, Y. (2019). The Ralstonia solanacearum effector RipN suppresses plant PAMP-triggered immunity, localizes to the endoplasmic reticulum and nucleus, and alters the $\mathrm{NADH} / \mathrm{NAD}(+)$ ratio in Arabidopsis. Mol. Plant Pathol. 20, 533-546. doi: 10.1111/mpp. 12773

Swiderski, M. R., Birker, D., and Jones, J. D. (2009). The TIR domain of TIR-NBLRR resistance proteins is a signaling domain involved in cell death induction. Mol. Plant Microbe Interact. 22, 157-165. doi: 10.1094/MPMI-22-2-0157

Takken, F. L., and Goverse, A. (2012). How to build a pathogen detector: structural basis of NB-LRR function. Curr. Opin. Plant Biol. 15, 375-384. doi: 10.1016/j. pbi.2012.05.001

Tang, D., Wang, G., and Zhou, J. M. (2017). Receptor kinases in plant-pathogen interactions: more than pattern recognition. Plant Cell 29, 618-637. doi: 10. 1105/tpc.16.00891

Tang, L., Qiu, L., Liu, C., Du, G., Mo, Z., Tang, X., et al. (2019). Transcriptomic insights into innate immunity responding to red rot disease in red alga Pyropia yezoensis. Int. J. Mol. Sci. 20:5970. doi: 10.3390/ijms20235970

Tanigaki, Y., Ito, K., Obuchi, Y., Kosaka, A., Yamato, K. T., Okanami, M., et al. (2014). Physcomitrella patens has kinase-LRR R gene homologs and interacting proteins. PLoS one 9:e95118. doi: 10.1371/journal.pone.0095118

Urbach, J. M., and Ausubel, F. M. (2017). The NBS-LRR architectures of plant R-proteins and metazoan NLRs evolved in independent events. Proc. Natl. Acad. Sci. U.S.A. 114, 1063-1068. doi: 10.1073/pnas.1619730114

Valkov, E., Stamp, A., Dimaio, F., Baker, D., Verstak, B., Roversi, P., et al. (2011). Crystal structure of Toll-like receptor adaptor MAL/TIRAP reveals the molecular basis for signal transduction and disease protection. Proc. Natl. Acad. Sci. U.S.A. 108, 14879-14884. doi: 10.1073/pnas.1104780108

Ve, T., Williams, S. J., and Kobe, B. (2015). Structure and function of Toll/interleukin-1 receptor/resistance protein (TIR) domains. Apoptosis 20, 250-261. doi: 10.1007/s10495-014-1064-2

Wagner, S., Stuttmann, J., Rietz, S., Guerois, R., Brunstein, E., Bautor, J., et al. (2013). Structural basis for signaling by exclusive EDS1 heteromeric complexes with SAG101 or PAD4 in plant innate immunity. Cell Host Microbe 14, 619-630. doi: 10.1016/j.chom.2013.11.006

Wan, L., Essuman, K., Anderson, R. G., Sasaki, Y., Monteiro, F., Chung, E. H., et al. (2019). TIR domains of plant immune receptors are $\mathrm{NAD}(+)$-cleaving enzymes that promote cell death. Science 365, 799-803. doi: 10.1126/science.aax1771

Wang, C., Zhang, X., and Mou, Z. (2016). Comparison of nicotinamide adenine dinucleotide phosphate-induced immune responses against biotrophic 
and necrotrophic pathogens in Arabidopsis thaliana. Plant Signal. Behav. 11:e1169358. doi: 10.1080/15592324.2016.1169358

Wang, J., Hu, M., Wang, J., Qi, J., Han, Z., Wang, G., et al. (2019a). Reconstitution and structure of a plant NLR resistosome conferring immunity. Science 364:eaav5870. doi: 10.1126/science.aav5870

Wang, J., Wang, J., Hu, M., Wu, S., Qi, J., Wang, G., et al. (2019b). Ligand-triggered allosteric ADP release primes a plant NLR complex. Science 364:eaav5868. doi: $10.1126 /$ science.aav5868

Williams, S. J., Sohn, K. H., Wan, L., Bernoux, M., Sarris, P. F., Segonzac, C., et al. (2014). Structural basis for assembly and function of a heterodimeric plant immune receptor. Science 344, 299-303. doi: 10.1126/science.1247357

Williams, S. J., Yin, L., Foley, G., Casey, L. W., Outram, M. A., Ericsson, D. J., et al. (2016). Structure and function of the TIR domain from the grape NLR protein RPV1. Front. Plant. Sci. 7:1850. doi: 10.3389/fpls.2016. 01850

Wu, Z., Li, M., Dong, O. X., Xia, S., Liang, W., Bao, Y., et al. (2019). Differential regulation of TNL-mediated immune signaling by redundant helper CNLs. New Phytol. 222, 938-953. doi: 10.1111/nph.15665

Xu, Y., Tao, X., Shen, B., Horng, T., Medzhitov, R., Manley, J. L., et al. (2000). Structural basis for signal transduction by the Toll/interleukin-1 receptor domains. Nature 408, 111-115. doi: 10.1038/35040600

Yue, J. X., Meyers, B. C., Chen, J. Q., Tian, D., and Yang, S. (2012). Tracing the origin and evolutionary history of plant nucleotide-binding site-leucine-rich repeat (NBS-LRR) genes. New Phytol. 193, 1049-1063. doi: 10.1111/j.14698137.2011.04006.x

Zhang, L., Chen, S., Ruan, J., Wu, J., Tong, A. B., Yin, Q., et al. (2015). Cryo-EM structure of the activated NAIP2-NLRC4 inflammasome reveals nucleated polymerization. Science 350, 404-409. doi: 10.1126/science. aac5789
Zhang, Q., Zmasek, C. M., Cai, X., and Godzik, A. (2011). TIR domain-containing adaptor SARM is a late addition to the ongoing microbe-host dialog. Dev. Comp. Immunol. 35, 461-468. doi: 10.1016/j.dci.2010.11.013

Zhang, X., Bernoux, M., Bentham, A. R., Newman, T. E., Ve, T., Casey, L. W., et al. (2017). Multiple functional self-association interfaces in plant TIR domains. Proc. Natl. Acad. Sci. U.S.A. 114, E2046-E2052. doi: 10.1073/pnas.16212 48114

Zhang, X., and Mou, Z. (2012). Expression of the human NAD(P)-metabolizing ectoenzyme CD38 compromises systemic acquired resistance in Arabidopsis. Mol. Plant Microbe Interact. 25, 1209-1218. doi: 10.1094/MPMI-10-11-0278

Zhao, T., Rui, L., Li, J., Nishimura, M. T., Vogel, J. P., Liu, N., et al. (2015). A truncated NLR protein, TIR-NBS2, is required for activated defense responses in the exo70B1 mutant. PLoS Genet. 11:e1004945. doi: 10.1371/journal.pgen. 1004945

Zhao, Z. Y., Xie, X. J., Li, W. H., Liu, J., Chen, Z., Zhang, B., et al. (2019). A CellPermeant Mimetic of NMN Activates SARM1 to Produce Cyclic ADP-Ribose and Induce Non-apoptotic Cell Death. iScience 15, 452-466. doi: 10.1016/j.isci. 2019.05.001

Conflict of Interest: The authors declare that the research was conducted in the absence of any commercial or financial relationships that could be construed as a potential conflict of interest.

Copyright $\odot 2020$ Bayless and Nishimura. This is an open-access article distributed under the terms of the Creative Commons Attribution License (CC BY). The use, distribution or reproduction in other forums is permitted, provided the original author(s) and the copyright owner(s) are credited and that the original publication in this journal is cited, in accordance with accepted academic practice. No use, distribution or reproduction is permitted which does not comply with these terms. 